\title{
Resource Allocation for Parallel Links in Hybrid WiFi/VLC Systems
}

\author{
A Thesis \\ Presented to \\ the faculty of the School of Engineering and Applied Science \\ University of Virginia
}

in partial fulfillment

of the requirements for the degree

Master of Science

by

Abdulmajeed Muflih A Alenezi

December 2017 


\section{APPROVAL SHEET}

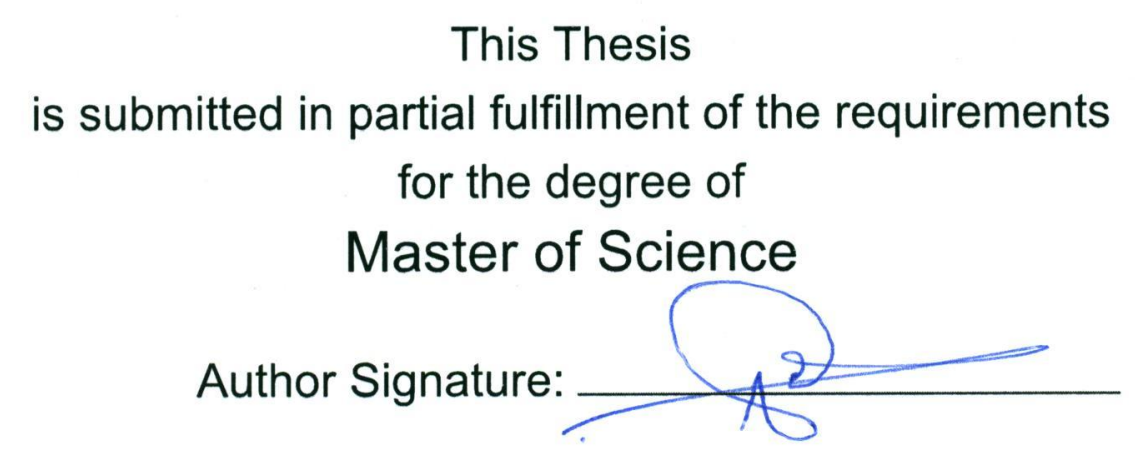

This Thesis has been read and approved by the examining committee:

Advisor: Prof. Maite Brandt-Pearce

Committee Member: Prof. Stephen G. Wilson

Committee Member: Prof. Andreas Beling

Committee Member:

Committee Member:

Committee Member:

Accepted for the School of Engineering and Applied Science:

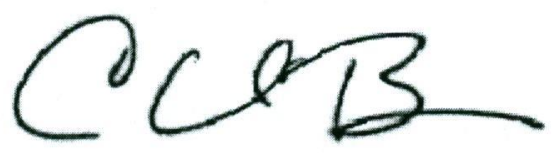

Craig H. Benson, School of Engineering and Applied Science

December 2017 


\section{Acknowledgements}

I would like to gratefully and sincerely thank my advisor, Professor Maite BrandtPearce for her guidance, understanding, patience, and most importantly, persistent encouragement and continuous support during my graduate studies at the University of Virginia. Her deep knowledge of communication theory and her impeccable attitude towards research have greatly helped me to form a rigorous, dedicated, and creative research style for my future career life in the communication engineering field.

It is a blessing to be surrounded by many colleagues and friends at the University of Virginia. I am grateful to all my friends in Charlottesville and for making my life at U.Va. enjoyable. I am thankful to my family for their patience, help, and love.

Finally, I'm grateful for the generosity of the Kingdom of Saudi Arabia's government in funding my graduate studies at the University of Virginia. 


\section{Abstract}

Due to the high demand for wireless data transmission recently, hybrid systems have been proposed to satisfy that demand. Visible light communication (VLC) is a promising approach due to many advantages such as high capacity, operating at an unregulated frequency band, and low power consumption. We propose a new hybrid system that simultaneously transmits data over two parallel links: RF and VLC. The uplink is set to be only RF while the downlink is a combination of two independent systems that transmit data in parallel without interference. The proposed system can offload users from one system to another to ensure achieving either a maximum or fair data rate over the users. The proposed model tested better than stand-alone WiFi, and two other hybrid systems. Simulations showed that it has the best performance in term of either maximizing the minimum data rate or maximizing the average data rate. 


\section{Contents}

1 Introduction 1

1.1 Background .......................... 1

1.2 Literature Review . . . . . . . . . . . . . . . . . . . . . . 4

1.3 Contribution and Thesis Outline . . . . . . . . . . . . . 7

2 System Description $\quad 8$

2.1 System Model . . . . . . . . . . . . . . . . . 8

$2.2 \mathrm{WiFi}$ Model . . . . . . . . . . . . . . . . . . 9

2.3 VLC Model . . . . . . . . . . . . . . . . . . . 11

2.3.1 Basic Principles of VLC . . . . . . . . . . . . . . 11

2.3.2 VLC Link Characteristics . . . . . . . . . . . . . . . 12

2.4 Modulation and Multiple Access Schemes . . . . . . . . . . . . . 17

2.4.1 Time Division Multiple Access (TDMA) . . . . . . . . . . . 17

2.4.2 Orthogonal Frequency Division Multiplexing (OFDM) . . . . 19

2.4.3 M-ary Pulse Amplitude Modulation (M-PAM) . . . . . . . . . 19

3 Optimization $\quad 22$

3.1 Problem Formulation . . . . . . . . . . . . . . . . . . 22

3.2 Problem Solution . . . . . . . . . . . . . . . . 24 
4 Numerical Results $\quad 28$

$4.1 \mathrm{WiFi}$ Only .......................... 29

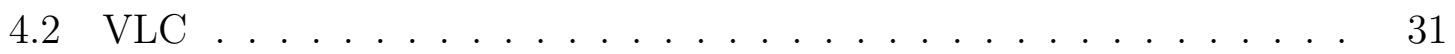

4.3 Hybrid VLC and WiFi . . . . . . . . . . . . . . . . . . . 32

4.3.1 Optimal Fair Allocation . . . . . . . . . . . . . . . . 35

4.3.2 Maximum Throughput Allocation . . . . . . . . . . . . . . . 40

4.3 .3 Case Study . . . . . . . . . . . . . . . . . 46

5 Conclusion and Future Work $\quad 51$

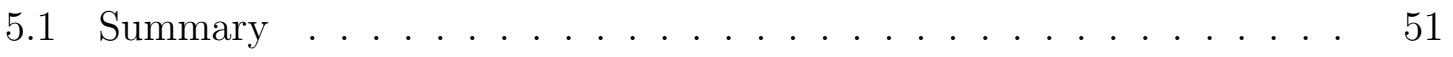

5.2 Conclusion . . . . . . . . . . . . . . . . . . . . 52

5.3 Future Work . . . . . . . . . . . . . . . . . 52 


\section{List of Figures}

1.1 Bandwidth density of $(a) \operatorname{RF}(b)$ VLC $\ldots \ldots \ldots \ldots$

2.1 The proposed hybrid VLC and RF model . . . . . . . . . . . 9

2.2 VLC location in the electromagnetic spectrum $[1] \ldots \ldots \ldots$

2.3 VLC block diagram $[2] \ldots \ldots \ldots$

2.4 25-LED lamp structure, (a) side view, (b) bottom view [3] $\ldots \ldots$.

2.5 Link configuration in $\operatorname{LOS} \ldots \ldots \ldots \ldots$

2.6 TDMA Frame Structure $[4] \ldots \ldots \ldots \ldots$

2.7 OFDM Signal Frequency Spectrum $[5] \ldots \ldots \ldots$

2.8 (a) The transmitted signal using 4-PAM (b) The original signal . . . 21

3.1 Airtime fairness between 2 users . . . . . . . . . . . . . . . . 23

4.1 Top view on the room to show the locations of the AP's $\ldots \ldots 28$

4.2 The relationship between data rate and distance for a WiFi system using the parameters in Table $4.1 \ldots \ldots \ldots \ldots$

4.3 The relationship between the data rate and transmitted power in RF system assuming the parameters in Table 4.1. for a user $1 \mathrm{~m}$ away

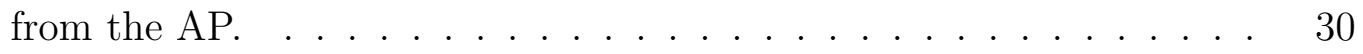

4.4 Received power distribution on the floor for the assumptions in Table

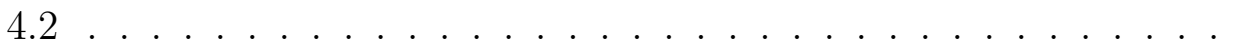


4.5 The relationship between the peak power of the LED and the SNR assuming the receiver is $3 \mathrm{~m}$ directly below the lamp. . . . . . . . . . 33

4.6 SINR coverage of VLC in a room of size $(8 \mathrm{~m} \times 8 \mathrm{~m} \times 3 \mathrm{~m}) \ldots . . . \quad 35$

4.7 Comparison between $\mathrm{WiFi}$ and the proposed hybrid system in term of minimum data rate for parameters listed in Tables 4.1 and 4.2. . . . 36

4.8 Comparison between the hybrid systems in term of the minimum data rate for parameters listed in Tables 4.1 and 4.2 assuming the VLC AP's do not experience co-channel interference . . . . . . . . . . . . 37

4.9 Comparison between the hybrid systems in term of the minimum data rate when the VLC AP's experience co-channel interference for parameters listed in Tables 4.1 and 4.2 . . . . . . . . . . . . . . . 38

4.10 SINR coverage of VLC in a room of size $(5 \mathrm{~m} \times 5 \mathrm{~m} \times 3 \mathrm{~m}) \ldots . . . \quad 38$

4.11 Comparison between the hybrid systems in term of the minimum data rate in the small room when the VLC AP's experience co-channel interference for parameters listed in Tables 4.1 and 4.2 . . . . . . . .

4.12 Comparison between the hybrid systems in term of the minimum data rate in the small room when the VLC AP's do not experience cochannel interference for parameters listed in Tables 4.1 and 4.2 . . .

4.13 Comparison between the proposed system and WiFi in term of the average data rate when the VLC AP's experience co-channel interference for parameters listed in Tables 4.1 and $4.2 \ldots$. . . . . . . . . .

4.14 Comparison between the proposed system and WiFi in term of the average data rate when the VLC AP's do not experience co-channel interference for parameters listed in Tables 4.1 and 4.2. . . . . . . . 
4.15 Comparison between all the systems in term of the average data rate when the VLC AP's experience co-channel interference for parameters listed in Tables 4.1 and $4.2 . \ldots \ldots \ldots \ldots$. . . . . . . . . .

4.16 Comparison between all the systems in term of the average data rate when the VLC AP's do not experience co-channel interference for parameters listed in Tables 4.1 and $4.2 \ldots \ldots \ldots \ldots$

4.17 Comparison between all the systems in term of the average data rate for the small room when the VLC AP's experience co-channel interference for parameters listed in Tables 4.1 and $4.2 \ldots$. . . . . . . . . .

4.18 Comparison between all the systems in term of the average data rate for the small room when the VLC AP's do not experience co-channel interference for parameters listed in Tables 4.1 and $4.2 . . . . . .$. 


\section{List of Tables}

2.1 Notations of VLC channel gain equation . . . . . . . . . . 13

3.1 All posibilities for a user to connect to the proposed hybrid system . 26

4.1 Parameters used in the RF Model . . . . . . . . . . . . . . . . . 29

4.2 Parameters used in the VLC Model . . . . . . . . . . . . . . . . 31

4.3 The maximum data rate based on distance for case $1 \ldots \ldots 47$

4.4 Comparison between the hybrid systems in term of minimum and av-

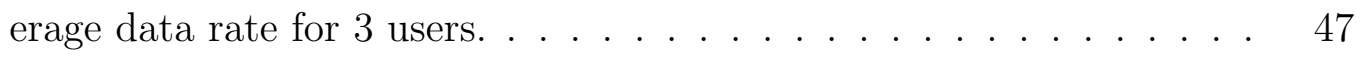

4.5 Calculated data rate in Mbps for all users from each AP's . . . . . 48

4.6 Indicator variable for each user when implementing the optimal fair allocation (Model (1)). . . . . . . . . . . . . . . . . . . 48

4.7 Indicator variables for each user when implementing the hybrid system where the user always connect to both RF and VLC (Model (3)). . . 49

4.8 Indicator variables for each user when implementing the hybrid system where the user connects to either the VLC or RF AP (Model (2)). . . 50

4.9 Comparison between the hybrid systems in term of minimum and average data rate for 7 users. . . . . . . . . . . . . 50

4.10 Indicator variables for each user when implementing the maximum

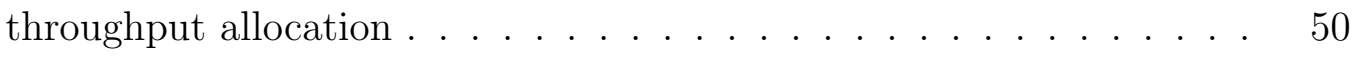




\section{Acronyms}

$\begin{array}{ll}\text { AP } & \text { Access point } \\ \text { BER } & \text { Bit error rate } \\ \text { CA } & \text { Collision avoidance } \\ \text { CDMA } & \text { Code-division multiple access } \\ \text { CSMA } & \text { Carrier-sense multiple access } \\ \text { FDM } & \text { Frequency-division multiplexing } \\ \text { FFT } & \text { Fast Fourier transform } \\ \text { FOV } & \text { Field of view } \\ \text { FSO } & \text { Free space optical } \\ \text { GN } & \text { Gaussian noise } \\ \text { IEEE } & \text { Institute of Electrical and Electronics Engineers } \\ \text { IM/DD } & \text { Intensity modulation and direct detection } \\ \text { LED } & \text { Light-emitting diode } \\ \text { LOS } & \text { Line of sight } \\ \text { LTE } & \text { Long term evoloving } \\ \text { MAC } & \text { Medium access control } \\ \text { M-PAM } & \text { M-ary pulse amplitude modulation } \\ \text { OFDM } & \text { Orthogonal frequency-division multiplexing } \\ \text { PLC } & \text { Power line communication } \\ \text { PSD } & \text { Power spectral density } \\ \text { PSK } & \text { Phase shift keying } \\ \text { QAM } & \text { Quadrature amplitude modulation } \\ \text { RAT } & \text { Radio access technologies } \\ \text { RF } & \text { Radio frequency } \\ \text { SINR } & \text { Signal to noise plus interference ratio } \\ \text { SNR } & \text { Signal to noise ratio } \\ \text { TDMA } & \text { Time Division multiplexing Access } \\ \text { VLC } & \text { Visible light communication } \\ \text { WiFi } & \text { Wireless fidelity } \\ \text { WWRF } & \text { Wireless World Research Forum } \\ & \end{array}$




\section{Chapter 1}

\section{Introduction}

Our aim in this chapter is to introduce the idea of hybrid communication systems and why we need these heterogeneous systems. We address this by describing the background of wireless networks, literature on the subject, and our contribution in solving the problem. We focus our attention on hybrid WiFi and visible light communication systems.

\section{$1.1 \quad$ Background}

Over the past years, researchers have tried to improve wireless networks to satisfy the high demand for data. Several studies suggest that with the high usage of devices that need to connect to a network, developers might not be able to improve the network speed to achieve their demands [6]. For example, the Wireless World Research Forum (WWRF) expects the number of wireless devices to reach 7 trillion by 2020 [7], which is much higher than the expected population in 2020. Most people now have at least two wireless devices, such as smart-phone, tablet, laptop or watch, that consume the wireless network capacity whether or not the user is actively using them. We propose a solution to the wireless problem based on coordinated use of 
$\mathrm{WiFi}$ and visible light communications.

The WiFi standards 802.11 have improved over the past years in many areas such as speed, range, and cost [8]. Starting from 802.11b, WiFi supported a maximum data rate of 11 Mbps. These standards improved to support up to 1 Gbps in 802.11ac, but still not enough to satisfy the demand for higher data rate.

Researchers have thought of new methods to track the high demand for data by using heterogeneous systems that allow the user to use two different technologies on the same device. To support the idea of heterogeneous systems, researchers developed multi-radio access technologies (RAT) that allow the user to connect to more than one system simultaneously or one system at a time [9]. One possible heterogeneous system would be the hybrid Long-Term Evolution (LTE) and WiFi, as most of the current devices support both systems. However, working on the same frequency band is challenging as each system has a different MAC layer. Not only that, but LTE is not designed to assume that the spectrum is shared with other systems, which creates interference when transmitting data. To solve these problems, developers need to implement more complicated schemes.

Another possible heterogeneous system, especially for indoor environments, is to use visible light communication (VLC), which is an optical wireless communication technology that uses the light to transmit the data. Instead of RF communications, VLC is a promising area of interest as it overcomes most of the disadvantages of the RF systems such as limited bandwidth and interference. It also has many advantages such as

- Low power consumption: LED lights are energy efficient and they are already consuming power anyway.

- Safe for humans: In VLC, the light source is modulated at high frequency that can be detected by receivers such as photodiodes without affecting human eyes 
as they operate at higher frequency than the human eyes can recognize. Unlike RF, light cannot penetrate the skin.

- High data rate: Several factors lead to higher data rate such as

- LED's can achieve high data rate by using multilevel amplitude modulation.

- The free use of the visible light spectrum.

- Small access areas lead to a high resource reuse rate.

VLC has a high capacity as each lamp can operate as an independent access point (AP). Figure 1.1 shows both RF and VLC channel densities when connecting to multiple users. In RF, all users share the $200 \mathrm{Mbps}$ while in VLC, each AP has its own channel capacity. The most significant advantage of VLC over other heterogeneous systems is operating at a different frequency band, which allows the two systems to transmit without any interference [10].

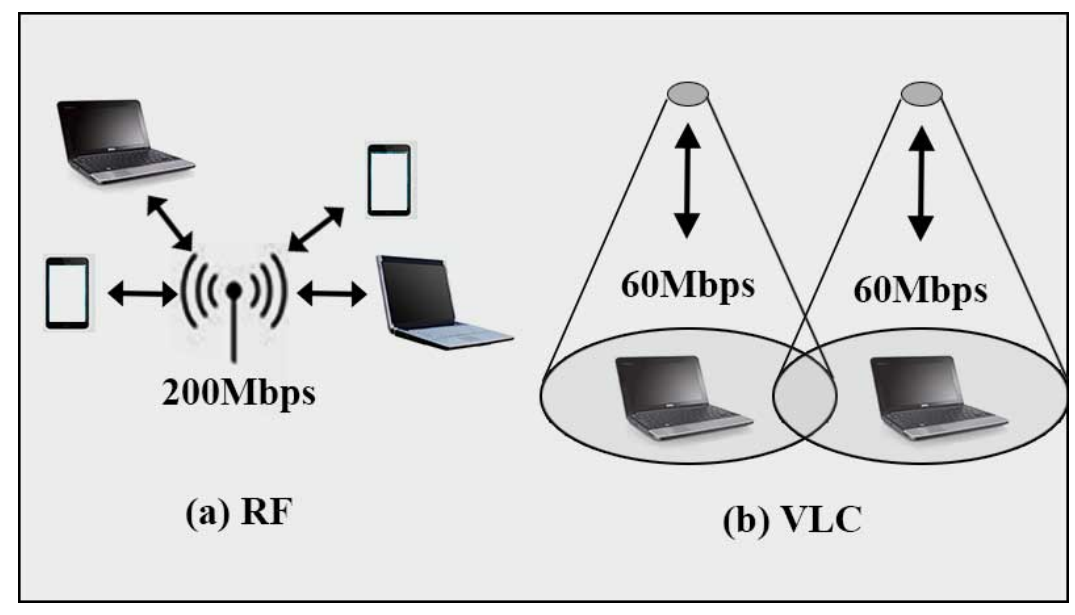

Figure 1.1: Bandwidth density of $(a)$ RF (b) VLC

As research in the field of VLC increases, several hybrid VLC/RF have been proposed over the past years. There have emerged two different approaches. One 
approach is to divide the hybrid system into WiFi for the uplink and VLC for the downlink. This approach is helpful as it is challenging to use VLC in the uplink, as you need to add a light source on the phone, which is not practical due to the movement of the users. The other approach is to use both systems as downlink but with different connection scenarios such as switching between the two systems or connecting to both systems at the same time. Alternatively, what we propose is to use WiFi for the uplink and both WiFi and VLC working in parallel for the downlink.

\subsection{Literature Review}

In [11], Byoung Hoon Jung et al. proposed a network-assisted user-centric WiFioffloading model that allows the user to offload from LTE to WiFi to achieve higher throughput in LTE-WiFi heterogeneous system. Their simulations showed $20 \%$ improvement in the per-user throughput after implementing their model. However, this research offloads LTE users to WiFi systems, which is not helpful in the case of an indoor environment where most of the users connect to the WiFi.

Similar to the previous study, Zhimin Li et al. published a study in 2016 about offloading user from LTE to WiFi using an multi-object optimization approach [12]. They considered the delay when offloading users from LTE to WiFi. Their simulation showed fair usage of the data when implementing their algorithm. As mentioned before, even though they achieved fairness among the users, offloading users to a crowded channel such as WiFi is not helpful in indoor environments as the capacity of the RF channel is not large.

In 2015, Shweta Sagari et al. published a paper regarding the interference characteristic of hybrid WiFi and LTE [13]. Several experiments were implemented to test the effect of LTE on WiFi signals. One study concluded that the WiFi signal is 
affected by the LTE as long as they are in the same range. The second study showed that the overall throughput is proportional to the network density. For lower network density, the overall throughput increases but it decreases as the network density increases. Their simulation showed that the WiFi's throughput degraded by up to $97 \%$ due to the interference from the LTE signal.

To avoid the problem of working on the same frequency band, researchers started to consider VLC begining in 2000. Researchers published several papers regarding the hybrid VLC-WiFi systems. Below some of their work is detailed.

Regarding fairness and throughput, Xuan Li et al. studied the load balancing problem in hybrid WiFi and VLC using two different algorithms: centralized and distributed resource-allocation algorithms [14]. In this study, the users connect only to either WiFi or VLC. After testing various VLC cell formation schemes, they showed that the hybrid system has higher average throughput and fairness.

Another way of studying the hybrid VLC and RF system is by designing a parallel MAC protocol as Wei Guo et al. did in 2015 [15]. Their studies focused on the network part more than the physical layer by designing a parallel transmission MAC protocol based on carrier-sense multiple access with a collision avoidance (CSMA/CA) algorithm and parallel transmission. The RF channel is used as a control channel while transmitting the data could be on both RF and VLC. When transmitting the data within VLC, the RF channel is only used as a control channel. By comparing the protocol with the traditional IEEE 802.11n protocol, they showed that the proposed design provides higher throughput and efficiency.

In 2014, Sihua Shao et al. published a paper about a hybrid VLC and WiFi system by using WiFi as an uplink channel and VLC as a downlink channel [16]. By using this method, they granted more capacity in the downlink channel than the standalone $\mathrm{WiFi}$, as the VLC has high capacity. They designed a solution for transmitting 
packets over two different channels: WiFi as uplink and VLC as downlink. Their results showed improvement in WiFi throughput in crowded areas of Internet users.

In 2016, Xu Bao et al. published a paper about a hybrid system using WiFi as an uplink channel and either WiFi or VLC as the downlink channel [17]. They presented some solutions for the VLC channel regarding handover and multi-user operation. Compared to the stand-alone WiFi, their results showed that the hybrid system has better throughput as the VLC provides high capacity. Unlike other research, they used VLC as a control channel. They also presented a protocol to solve the issue of both vertical and horizontal handover, which occurs when the user switches from a VLC AP to another AP or from a VLC AP to a WiFi AP.

In 2015, Mohamed Kashef et al. published research about a hybrid system using power line communication (PLC) in cascade with VLC and WiFi [18]. In this study, the user can receive data in parallel from both WiFi and VLC. They focused on the power consumption part of the hybrid system by maximizing the throughput over received power. For the same amount of transmitted power, their research showed that the hybrid system has better data rate compared to a stand-alone RF system.

In 2012, Yi Tang et al. published a paper about hybrid free space optical (FSO) and RF systems [19]. In this study, an algorithm that maximizes the throughput over two parallel links is designed. They show that the average throughput can be increased by adapting the encoder rate, symbol rate and modulation. Their results are better than the non adaptive designs, and similar to the use of adaptive schemes that are affected by outage conditions.

Among all these studies, no one proposed a system that supports both low and high density users environments. By offloading users from one AP to another, we can achieve fair or maximum usage of the hybrid system for all users. To ensure fairness, the proposed system can offload users not only from one system to another but also 
between the VLC AP's. In this way, we can achieve the maximum possible fairness or throughput that the hybrid system is capable of.

\subsection{Contribution and Thesis Outline}

The main contribution of this thesis is to develop a new hybrid WiFi/VLC that uses $\mathrm{WiFi}$ as the uplink and allows the user to receive data simultaneously from two parallel independent communication links, the WiFi and VLC. The proposed system tests two options:

- a fair option where the highest minimum data rate is found.

- a highest throughput option where the maximum total data rate is found.

To do that, the proposed system runs through all possible scenarios and find the best solution for each option.

The thesis is divided into five chapters. The proposed system is explained in details in Chapters 2 and 3. In Chapter 2, the proposed system is explained in details. Each system is explained with the modulation or multiple access schemes used. The main contribution of the author is addressed in Chapter 3. In Chapter 3, we formulate the problem and propose a solution to achieve the best results. Chapter 4 shows some numerical results and simulations for the proposed system. Finally, we conclude the thesis in Chapter 5 and explain some future work that can be performed. 


\section{Chapter 2}

\section{System Description}

In this chapter, the proposed hybrid system model is described in detail. Both VLC and WiFi systems are illustrated. The second section explains how to calculate the data rate for each system. Finally, the modulations and multiple access schemes that we use are explained in the last section.

\subsection{System Model}

In this section, we illustrate the proposed hybrid model. The model consists of two independent parallel communications links. For the uplink scenario, the data is transmitted through only the WiFi link. This approach is confirmed to be the best practical case in hybrid systems since the uplink typically requires a lower data rate. For the downlink scenario, the proposed system can send data simultaneously using two independent parallel links: RF and VLC. The data can be sent simultaneously without interference from the other link. The receiver is designed with multiple wireless interfaces to receive the data from both links at the same time. Each user has an RF transceiver and a photodetector. Figure 2.1 illustrates the hybrid model. 


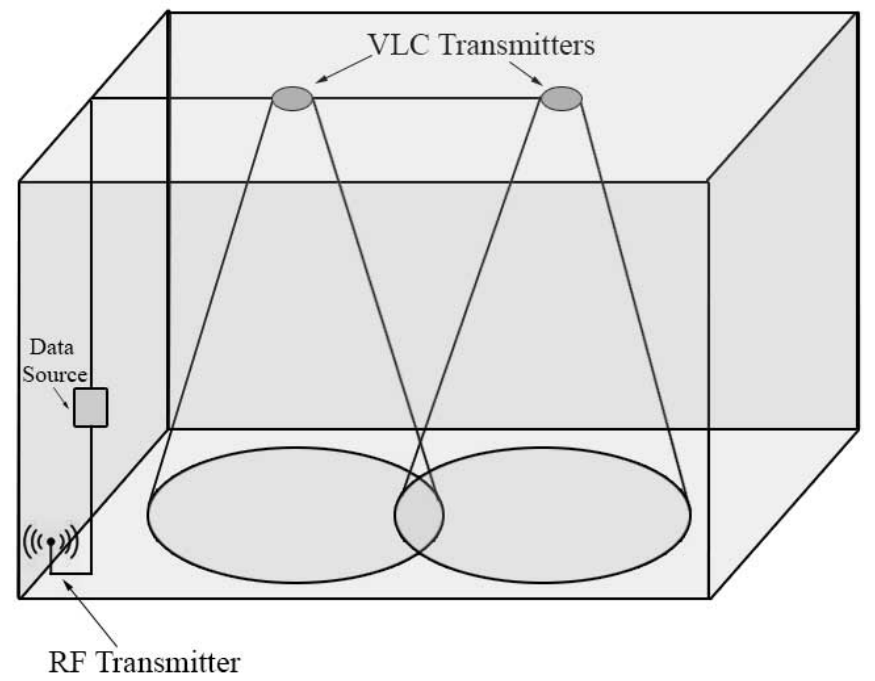

Figure 2.1: The proposed hybrid VLC and RF model

\section{$2.2 \quad$ WiFi Model}

In this study, we use the IEEE 802.11n standard for the WiFi model. In RF communications, it is important to note that the received signal depends on various factors such as the propagation loss, channel gain, and distance. The propagation loss $L\left(d_{R F}^{(k)}\right)$ as a function of the distance between the WiFi transmitter and user $k$ can be calculated using the following model [20]:

$$
L\left(d_{R F}^{(k)}\right)=\left\{\begin{array}{ll}
\left(\frac{4 d_{1} \pi}{\lambda}\right)^{2}\left(d_{R F}^{(k)} / d_{1}\right)^{2} & d_{R F}^{(k)} \leq d_{1} \\
\left(\frac{4 d_{1} \pi}{\lambda}\right)^{2}\left(d_{R F}^{(k)} / d_{1}\right)^{v} & d_{R F}^{(k)}>d_{1}
\end{array},\right.
$$

where $\lambda$ is the wavelength, $v$ is the critical parameter of the loss model, and $d_{1}$ is the reference distance. In a free space model which is assumed to be less than 8 meters, $v$ can be set to 2 , while $v$ is often set to around 3.3 for larger distances [20]. 
The received signal $P_{r R F}^{(k)}$ at a user location can be calculated using the following equation:

$$
P_{r R F}^{(k)}=\frac{P_{t R F}}{\left.L\left(d_{R F}^{(k)}\right)\right)}
$$

where $P_{t R F}$ is the transmitted signal of the WiFi router. By using the Shannon theorem, the data rate $R_{R F}^{(k)}$ for user $k$ can be approximated using the following equation:

$$
R_{R F}^{(k)}=W_{R F} * \log _{2}\left(1+\frac{P_{r R F}^{(k)}}{W_{R F} * N_{0 R F}}\right)
$$

where $N_{0 R F}$ is the noise spectral density, which is limited to thermal noise in this study, and $W_{R F}$ is the bandwidth of the WiFi channel. Algorithm 1 summarizes the steps of calculating the data rate in the WiFi model.

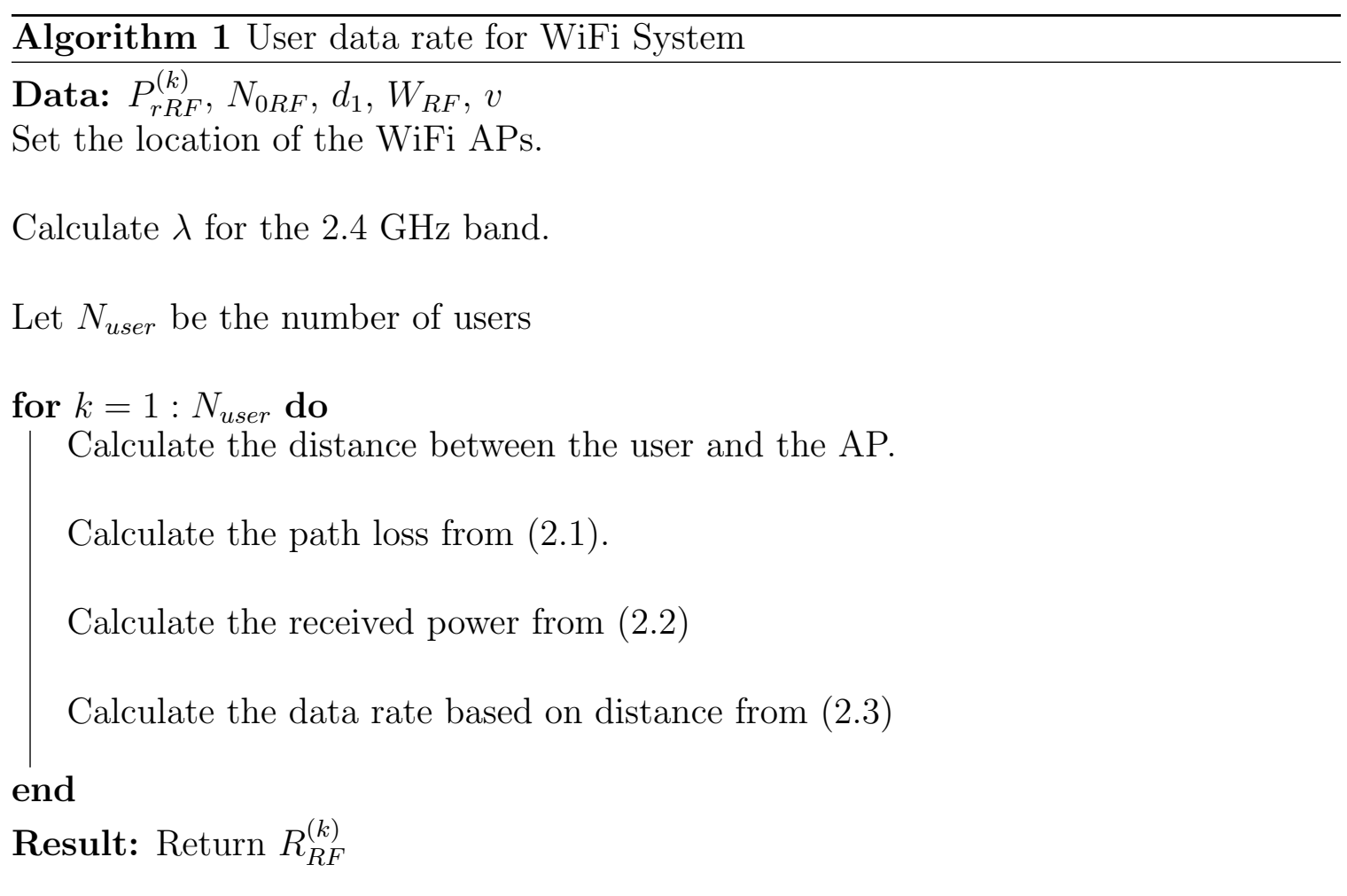




\subsection{VLC Model}

In this section, we illustrate the VLC model. We start by analyzing the basic principles of VLC, the link characteristics, and how to calculate the data rate.

\subsubsection{Basic Principles of VLC}

Visible light communication is a new data communication that uses the visible light between 400 and $800 \mathrm{THz}$ to transmit the data. As shown in Figure 2.2, VLC works at high frequency which makes it a perfect system to work alongside the RF systems without interference.

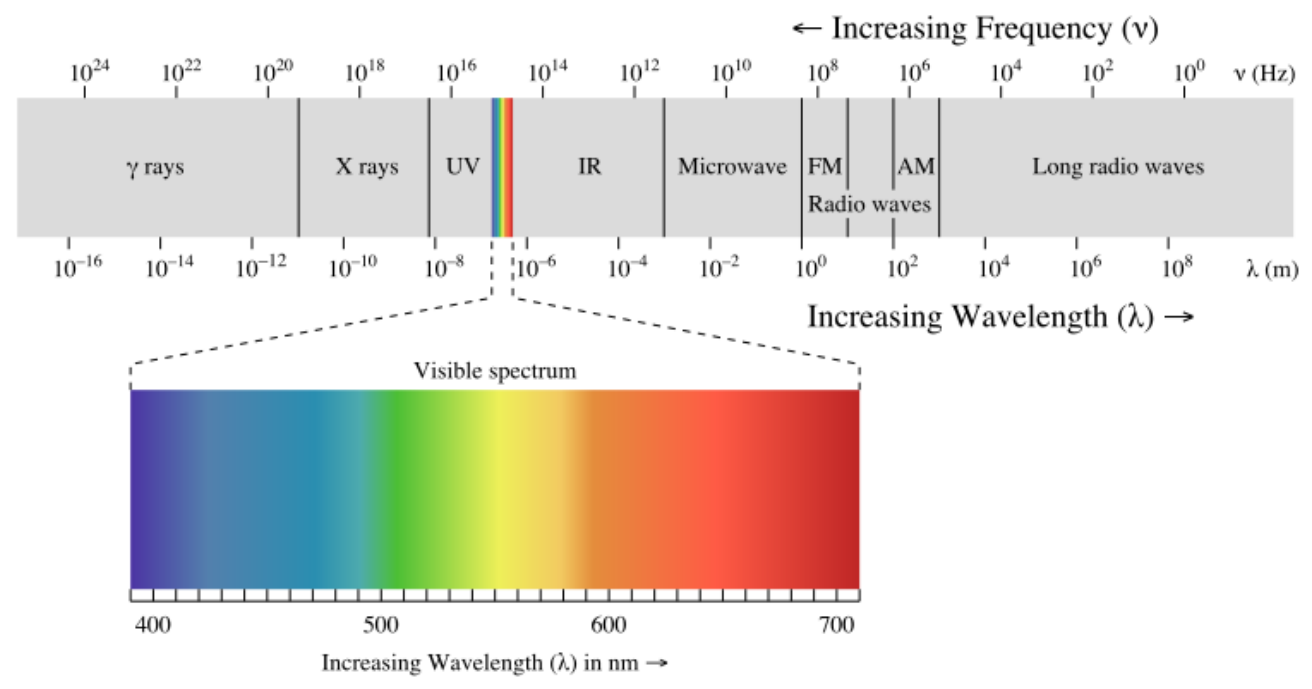

Figure 2.2: VLC location in the electromagnetic spectrum [1]

In VLC, the data is transmitted by a light source. Switching the light on is similar to transmitting a 'one' and likewise switching the light off means transmitting a 'zero'. For the VLC to work efficiently, we need a light source that can switch on and off as fast as possible. LED light bulbs are widely used in VLC due to their ability to switch the lamp at very high speeds [21]. When the signal is transmitted, a photodetector 
at the receiver is used to detect the light and convert it to a photocurrent. Figure 2.3 shows the block diagram of the VLC system.

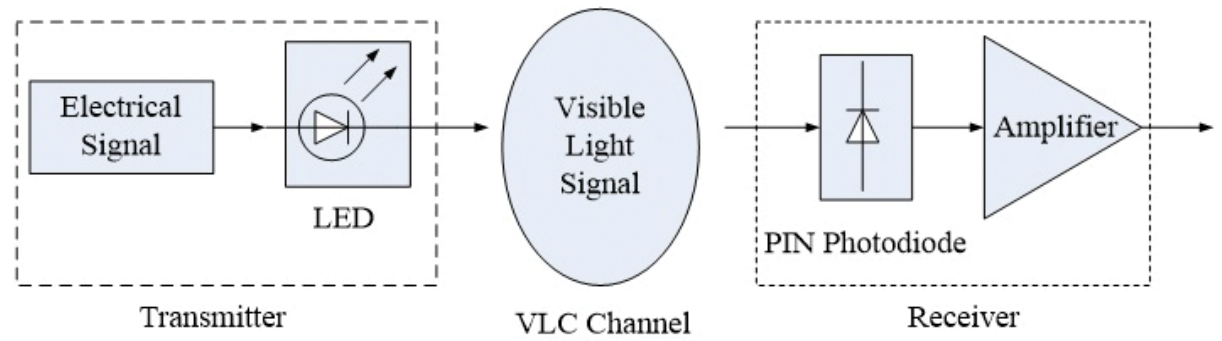

Figure 2.3: VLC block diagram [2]

\subsubsection{VLC Link Characteristics}

There are two types of links for indoor environments: the direct line of sight (LOS), and non-line of sight (NLOS). In this study, we consider only the LOS. In LOS, having a narrow field of view (FOV) on both the transmitter and receiver is essential. [22].

In the VLC model, it is essential to understand both the transmitter and the receiver. In the transmitter, we use an LED light source, which is proven to be one of the best light sources that can be used in VLC systems due to several factors as discussed in Chapter 1.

For the light source, there are many lamp models that illuminate all regions of the indoor space. To get the most benefit, we consider the model shown in Figure 2.4 [3]. The lamp consists of multiple LEDs divided into three layers. All LEDs are pointing toward the floor with different angles but the same transmitted power $P_{t L E D}$. For a given user, the line-of-sight (LOS) channel gain between the qth LED and the photodetector for user $k$ is given by the following equation:

$$
h_{q k}=\frac{A \cos (\phi)}{2 \pi d_{q k}^{2}}(m+1) \cos ^{m}(\psi), \quad \psi<F O V
$$


Note that $\phi$ is the angle between $\overrightarrow{r_{q k}}$ and $\overrightarrow{n_{k}}$ vectors, and $\psi$ is the angle between $\overrightarrow{r_{q k}}$ and $\overrightarrow{l_{q}}$ vectors. Table 2.1 summarizes the notations of the channel gain equation, while Figure 2.5 illustrates the vectors in (2.4). $m$ is the order of the Lambertian mode, which can be found from the LED's semi-angle at half power by using the following equation:

$$
m=\frac{\ln 2}{\ln \left(\cos \Phi_{1 / 2}\right)} .
$$

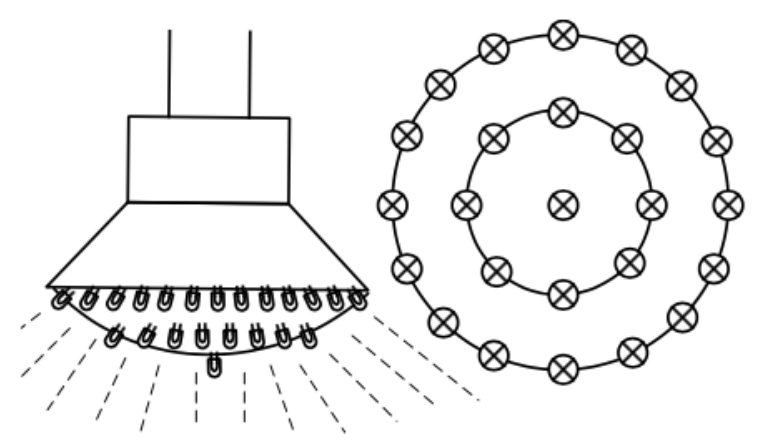

(a)

(b)

Figure 2.4: 25-LED lamp structure, (a) side view, (b) bottom view [3]

Table 2.1: Notations of VLC channel gain equation

\begin{tabular}{cc}
\hline Transmitted power from VLC-AP & $P_{t V L C}$ \\
Unit vector pointing toward receiver $k$ from LED $q$ & $\overrightarrow{r_{q k}}$ \\
Radiation unit direction vector for LED $q$ & $\overrightarrow{l_{q}}$ \\
Normal unit vector of the $k$ th receiver & $\overrightarrow{n_{k}}$ \\
Area of the photodetector & $A$ \\
Lambertian mode of the light source & $m$ \\
Distance between the transmitter $q$ and the receiver $k$ & $d_{q k}$ \\
Coverage angle of the photodetector & $F O V$ \\
\hline
\end{tabular}




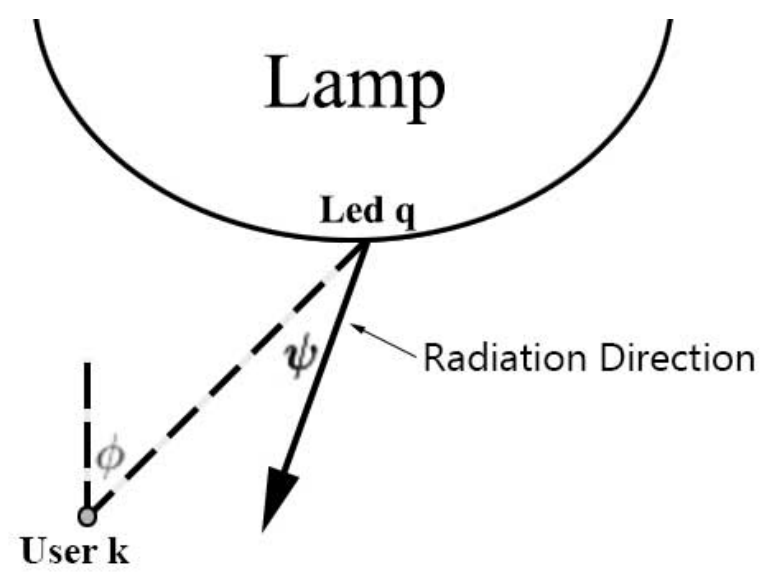

Figure 2.5: Link configuration in LOS

The received power for user $k$ from the VLC AP is calculated using the following equation:

$$
P_{r V L C}^{(k)}=\left(\sum_{q} h_{k q}\right) * P_{t V L C} .
$$

where $P_{t V L C}$ is the transmitted power from the VLC-AP.

To calculate the signal to noise ratio (SNR), we need to convert the optical power, $P_{r V L C}^{(k)}$, to electrical power, $\left(R e * P_{r V L C}^{(k)}\right)^{2}$, where $R e$ is the responsivity of the photodetector at the receiver. The $S N R$ can be calculated using the following equations:

$$
S N R_{V L C}^{(k)}=\frac{\left(R e * P_{r V L C}^{(k)}\right)^{2}}{N_{0 V L C} * W_{V L C}}
$$

Note that $W_{V L C}$ is the bandwidth of the VLC channel, and $N_{0 V L C}$ is the noise spectral density. The total noise affecting the VLC channel is a combination of thermal and shot noise. In the daytime, the shot noise dominates over the thermal noise. In the absence of background light, the thermal noise dominates over all noise [23]. $N_{0 V L C}$ can be calculated using the following equation 


$$
\begin{aligned}
& N_{0 V L C}=N_{\text {shot }}+N_{\text {thermal }} \\
& N_{0 V L C}=\left(2 q_{e} I W_{V L C}\right)+\left(\frac{4 K T W_{V L C} N_{\text {circuit }}}{\text { Resistance }}\right)
\end{aligned}
$$

$q_{e}$ is the electronic charge, $I$ is the photocurrent, which is related to $P_{r V L C}, K$ is Boltzmann's constant, $T$ is the room temperature, and $N_{\text {circuit }}$ is the circuit noise factor. Note that the photocurrent can be calculated using the following equation:

$$
I=R e * P_{r V L C}
$$

The only interference that needs to be considered is the co-channel interference between the VLC-AP's. If the user is located in an area covered by two VLC-AP's, there will be interference. To avoid that, we need to consider the signal to noise plus interference ratio (SINR), which for two VLC AP's can be calculated using the following equation:

$$
S I N R_{V L C 1}^{(k)}=\frac{\left(R e * P_{r V L C 1}^{(k)}\right)^{2}}{N_{0 V L C} * W_{V L C}+\left(R e * P_{r V L C 2}^{(k)}\right)^{2}}
$$

where $S I N R_{V L C 1}^{(k)}$ is the SINR at the first VLC AP. In all further expressions, we use the term SINR, that can be interpreted as SNR when the system experiences no interference. $P_{r V L C 1}^{(k)}$ is the received power from the same $\mathrm{AP}$ and $P_{r V L C 2}^{(k)}$ is the received power from the other AP's. For a user to be connected to a VLC's AP, the $S I N R$ needs to satisfy the equation below

$$
S I N R_{V L C}^{(k)} \geq S I N R_{\min }
$$

where $S I N R_{\min }$ is the minimum allowed $S I N R$ to connect to the VLC AP. SIN $R_{\text {min }}$ 
is calculated using the maximum allowed bit error rate (BER) when the modulation constellation size is 2 .

Once we get the $S I N R$ for each user, we need to choose an efficient M-ary modulation scheme. In this study, we used M-ary pulse amplitude modulation (M-PAM) as in [24]. In this scheme, the constellation size varies from one user to another based on their channel downlink quality. By using M-PAM, the bit error rate (BER) for any user $k$ can be calculated using the following formula [24]:

$$
B E R_{k}=\frac{M_{k}-1}{M_{k} \log _{2}\left(M_{k}\right)} \operatorname{erfc}\left(\sqrt{\frac{S I N R}{\left(M_{k}-1\right)^{2}}}\right)
$$

where $M_{k}$ is the modulation constellation size for user $k$, and erfc(.) is the complementary error function that can be defined as

$$
\operatorname{erfc}(x)=\frac{2}{\sqrt{\pi}} \int_{x}^{\infty} e^{-u^{2}} d u
$$

By setting a maximum BER, we can get the maximum modulation constellation size $\left(M_{k}\right)$ that satisfies $(2.13)$ such that the $B E R$ is less than or equal to $B E R_{\text {min }}$. Thus, assuming the symbol rate for all users to be the same, we can calculate the bit rate for a given $M_{k}$ using the following equation

$$
R_{V L C}^{(k)}=W_{V L C} * \log _{2}\left(M_{k}\right)
$$

This model is applied to all VLC AP's that a user can connect to. Algorithm 2.6 summarizes the VLC model. 


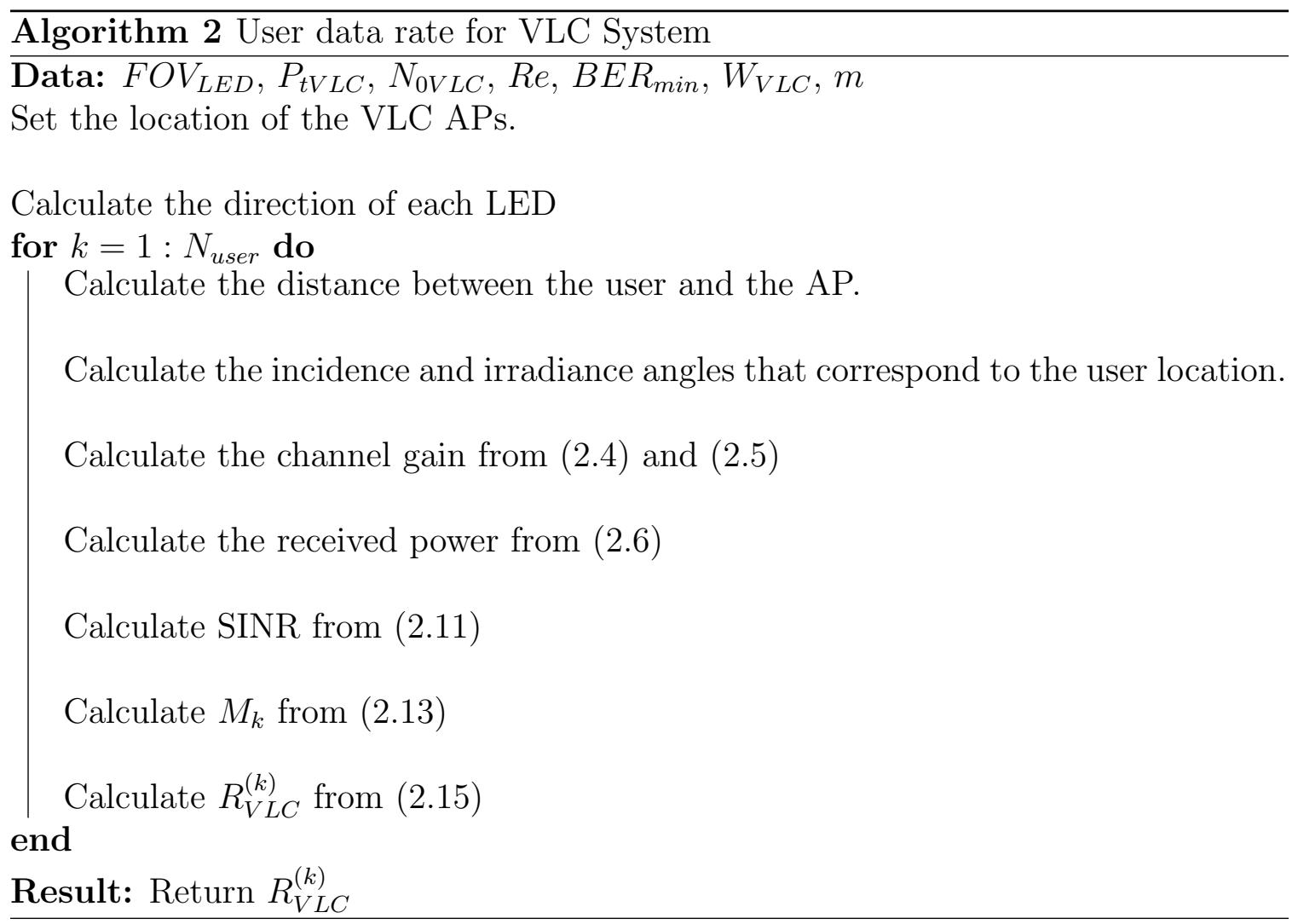

\subsection{Modulation and Multiple Access Schemes}

In this section, we discuss some of the modulations and multiple access schemes that can be used in the proposed hybrid system, and why we have chosen them.

\subsubsection{Time Division Multiple Access (TDMA)}

TDMA is a type of multiplexing used for a shared medium. Based on the number of users, the signal is divided into time slots where each user has a time slot to transmit the data. This process is repeated once all users transmit their data, which allows multiple users to share the same frequency channel. Figure 2.6 shows the frame structure and how the signal is divided into frames. These frames are divided into time slots where each user is allocated to one of them [25]. 


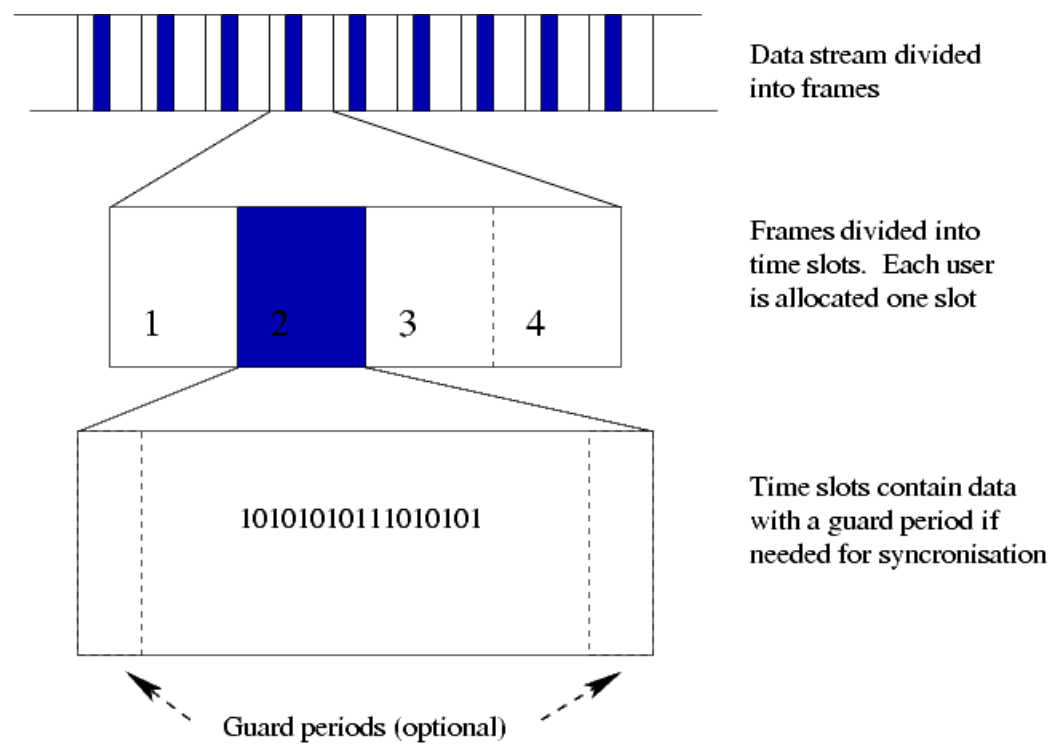

Figure 2.6: TDMA Frame Structure [4]

Some of the characteristics of the TDMA are listed below:

- Users share a single carrier frequency, or a multicarrier modulation such as OFDM

- Higher synchronization overhead compared to CDMA.

- Time slots can be assigned based on the demand in dynamic TDMA.

- Require less power control than CDMA.

- The transmissions do not create any interference among users assigned to the same AP.

In the WiFi model, we assume TDMA is used for airtime fairness since it agrees with the IEEE 802.11n standard. 


\subsubsection{Orthogonal Frequency Division Multiplexing (OFDM)}

OFDM is a scheme used for encoding data on multiple carrier frequencies. The data are carried by orthogonally-spaced sub-carrier signals on parallel channels. These sub-carriers are modulated separately using conventional modulation such as phase shift keying (PSK) or quadrature amplitude modulation (QAM) at a low sample rate. As shown in Figure 2.7, each sub-carrier is transmitted as a sinc function in frequency with overlapping side lobes that do not cause interference because the sub-channels are orthogonal. To recover the signal, the receiver multiplies the received signal by the sinusoids and recover the exact data. Alternatively, the receiver can use an FFT to process the signal. By using orthogonal sub-carriers, we can have more sub-carriers in the bandwidth compared with frequency division multiplexing (FDM), which increase the spectral efficiency [25].

OFDM is used in many applications especially in wireless communications such as IEEE 802.11g, n, and ac.

The main advantages of OFDM are:

- High spectral efficiency.

- Robust against co-channel interference and intersymbol interference.

- Low sensitivity to synchronization errors.

- Does not required tuned sub-channel receiver filters

\subsubsection{M-ary Pulse Amplitude Modulation (M-PAM)}

As the visible light in VLC is incoherent, we need to use intensity modulation and direct detection (IM/DD). In optical communication, one of the widely used 


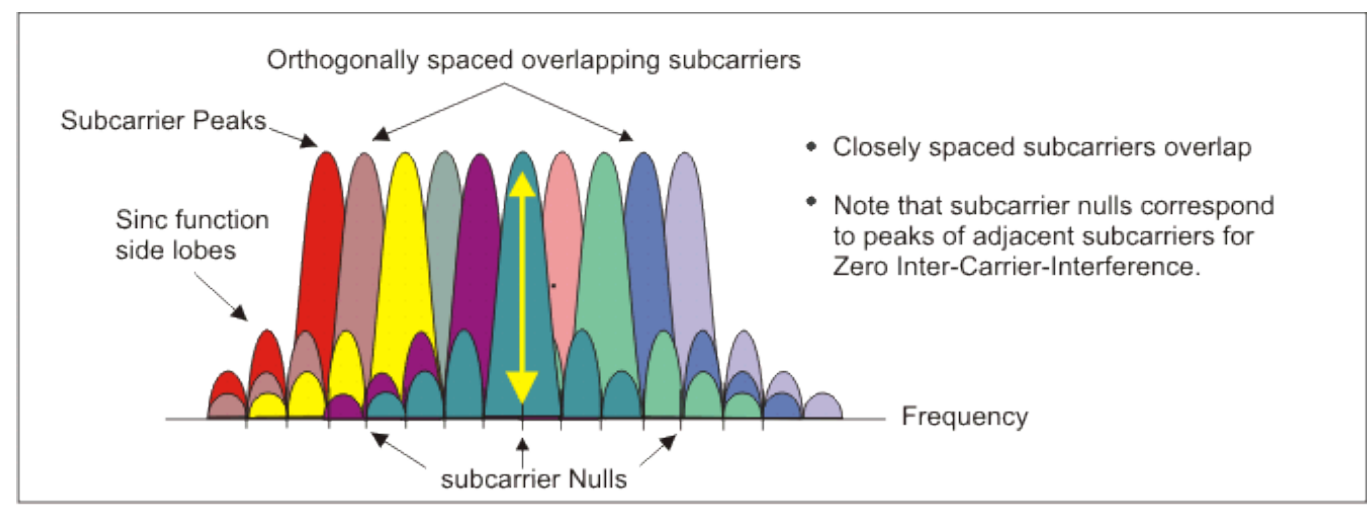

OFDM Signal Frequency Spectra

Figure 2.7: OFDM Signal Frequency Spectrum [5]

schemes is M-ary pulse amplitude modulation (MPAM) due to the simplicity of the transmitter and receiver structures [24].

In M-ary PAM, each group of $N$-bits is transmitted by a $M=2^{N}$ level pulse. We divide the data into a group of bits. For example, in 4-PAM, any transmitted signal stream is divided into pairs, i.e., '00' '01' '10' '11'. These four groups are transmitted as pulses with amplitude $\left(A_{n}\right)$ of $0,1,2$, and 3 respectively. To illustrate the MPAM, let's assume we want to transmit the data '0110111000111001'. In 4-PAM, the transmitted signal is divided into '01' '10' '11' '10' '00' '11' '10' '01' with amplitude of $1,2,3,2,0,3,2,1$, respectively, as shown in Figure 2.8. The modulated signal using M-PAM can be written as

$$
x(t)=\sum_{n=0}^{\infty} A_{n} G\left(t-T_{c}\right)
$$

where $G(t)$ is a pulse shape function.

One significant criterion in M-PAM that needs to be discussed is the bandwidth efficiency. Bandwidth efficiency is the information rate that can be transmitted over a given bandwidth in a specific communication system. It can be defined ideally as 


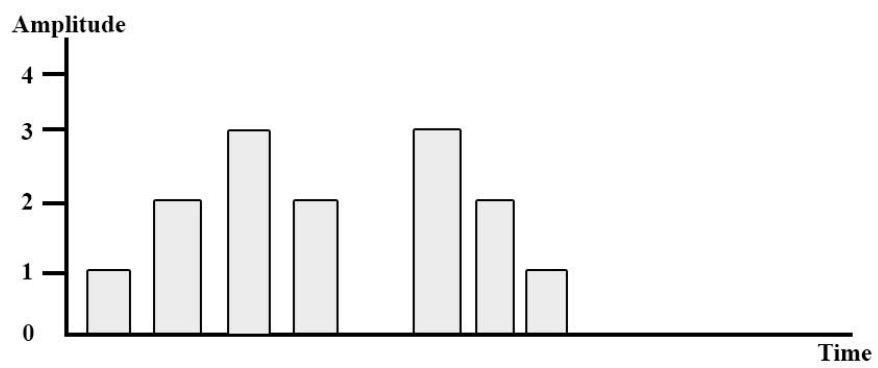

(a)

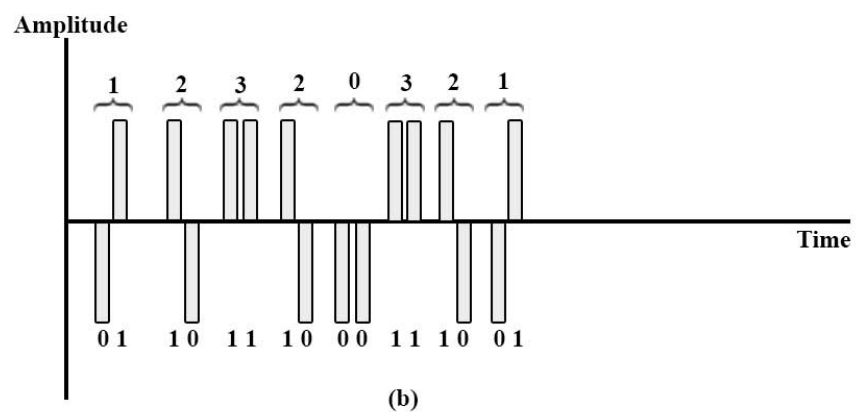

Figure 2.8: (a) The transmitted signal using 4-PAM (b) The original signal

$$
\eta_{B}=\frac{R_{b}}{W}=\log _{2}(M)
$$

where $R_{b}$ is the data rate, $W$ is the bandwidth and $M$ is the constellation modulation. We chose to use unipolar M-PAM for our VLC system because it has been shown to be more spectrally efficient and have a better performance than optical forms of OFDM in IM/DD systems. 


\section{Chapter 3}

\section{Optimization}

In this chapter, we describe the current problem in the WiFi system (the low data rate due to resource sharing), and why we need to use a hybrid system to overcome this issue. To do that, we first formulate the problem of summing the data rate of two different links. Then, we propose the solution to achieve the best possible results.

\subsection{Problem Formulation}

To begin, it is essential to understand how WiFi works in term of airtime fairness. Airtime fairness is a new technology implemented in WiFi lately to ensure each user receives fair time regardless of their data rate or the amount of data they need to transmit. For example, suppose two users are connected to the same WiFi AP with different data rate. Note that some users might use an old WiFi standard such $802.11 \mathrm{~g}$, while others use $802.11 \mathrm{n}$. There would be a significantly different data rate among these users. So assuming user 1 has a data rate of $20 \mathrm{Mbps}$, while user 2 has a data rate of 5 Mbps, user 1 will transmit 4 packets for the same amount of time that user 2 transmit 1 packet, as shown in Figure 3.1, due to the airtime fairness. 


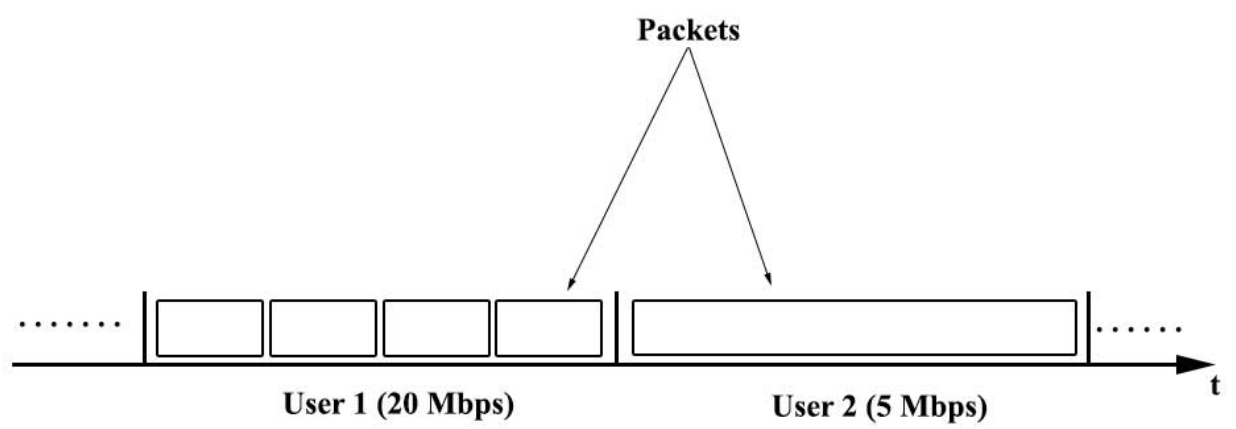

Figure 3.1: Airtime fairness between 2 users

As the time is divided equally, a user with low data rate will transmit some of its data and wait for other users to transmit at other times. Once user 1 finishes transmitting its data, user 2 will transmit all of its data without waiting. Thus, offloading users with low data rate to another network to give the users more time to transmit without affecting the other users' throughput needs to be considered.

For any user $k$ that is in the coverage of the hybrid RF/VLC system, there are three possible scenarios, which are:

- The user connects only to the WiFi link.

- The user connects only to a VLC link.

- The user connects to both VLC/WiFi links.

It is important to understand that connecting to both links is not always the best case in terms of fairness among all users. For example, suppose there are two users in the room. One user is covered by both systems, and only WiFi covers the other user. When user 1 connects to both systems, it is not fair for user 2 as it shares the capacity of the RF channel while user 1 uses both VLC and RF channels to receive 
data. Not only choosing between the two links but also which VLC AP should the user connect to is the goal of this study, as the user might connect to only one VLC AP even though it might be in the coverage of many AP's. Thus, summing the data rates of both links directly does not solve our problem.

Naively, one could expect that the data rate for user $k$ would be the sum of the data rates of the two parallel links

$$
R^{(k)}=R_{R F}^{(k)}+R_{V L C}^{(k)}
$$

where $R_{R F}^{(k)}$ is the data rate of the RF link as a function of SINR, and $R_{V L C}^{(k)}$ is the data rate of the VLC link as a function of SINR. Several studies solved the problem by optimizing over the received power of each link. However, this does not solve the problem as connecting to the best link's strength is not always the best solution, as we show below.

\subsection{Problem Solution}

To solve the problem, (3.1) needs to be modified to specify which AP can the user connect to. To do that, we need to add indicator variables for each AP and each user. These indicators are either 0 or 1 . When the indicator equals 0 , this means the user is not connected to the AP. Similarly, when the indicator equals 1, the user can connect to the AP. For the VLC model, the data rate for a user $k$ who is served by one AP can be calculated as

$$
R_{V L C}^{(k)}=\frac{R_{V L C} * I_{V L C}^{(k)}}{\sum_{n=1}^{N} I_{V L C}^{(n)}}
$$


where the sum of $I_{V L C}^{(n)}$ is the total number of users connected to the same AP and $N$ is the total number of users. Similarly, for the WiFi link, the data rate for user $k$ can be calculated as

$$
R_{R F}^{(k)}=\frac{R_{R F} * I_{R F}^{(k)}}{\sum_{n=1}^{N} I_{R F}^{(n)}}
$$

where $I_{R F}^{(k)}$ is the indicator for user $k$ to be connected to the RF link. Combining (3.2), (3.3), and (3.1), we can calculate the data rate for user $k$ in the proposed system as

$$
R^{(k)}=\frac{R_{R F} * I_{R F}^{(k)}}{\sum_{n=1}^{N} I_{R F}^{(n)}}+\frac{R_{V L C} * I_{V L C}^{(k)}}{\sum_{n=1}^{N} I_{V L C}^{(n)}}
$$

For $L$ number of VLC AP's, while the user can connect to only one VLC AP, (3.4) can be expanded to

$$
R^{(k)}=\frac{\left(R_{R F}^{(k)} * I_{R F}^{(k)}\right)}{\sum_{n=1}^{N} I_{R F}^{(n)}}+\frac{\left(R_{V L C 1}^{(k)} * I_{V L C 1}^{(k)}\right)}{\sum_{n=1}^{N} I_{V L C 1}^{(n)}}+\ldots+\frac{\left(R_{V L C L}^{(k)} * I_{V L C L}^{(k)}\right)}{\sum_{n=1}^{N} I_{V L C L}^{(n)}}
$$

where $L$ is the number of the VLC AP's in the system.

The optimization problem can be written as follows:

- Optimal fair allocation:

$$
\max _{I_{R F}, I_{V L C}} \min _{k}\left(\frac{\left(R_{R F}^{(k)} * I_{R F}^{(k)}\right)}{\sum_{n=1}^{N} I_{R F}^{(n)}}+\frac{\left(R_{V L C 1}^{(k)} * I_{V L C 1}^{(k)}\right)}{\sum_{n=1}^{N} I_{V L C 1}^{(n)}}+\ldots+\frac{\left(R_{V L C L}^{(k)} * I_{V L C L}^{(k)}\right)}{\sum_{n=1}^{N} I_{V L C L}^{(n)}}\right)
$$

- Maximum throughput allocation:

$$
\max _{I_{R F}, I_{V L C}} \operatorname{mean}\left(\frac{\left(R_{R F}^{(k)} * I_{R F}^{(k)}\right)}{\sum_{n=1}^{N} I_{R F}^{(n)}}+\frac{\left(R_{V L C 1}^{(k)} * I_{V L C 1}^{(k)}\right)}{\sum_{n=1}^{N} I_{V L C 1}^{(n)}}+\ldots+\frac{\left(R_{V L C L}^{(k)} * I_{V L C L}^{(k)}\right)}{\sum_{n=1}^{N} I_{V L C L}^{(n)}}\right)
$$

where $I_{V L C 1}^{(k)}, \ldots, I_{V L C L}^{(k)}$ are the indication function for each VLC AP for user $k$. To allow the user to connect to only one AP, the model needs to satisfy the following 
additional constraint:

$$
\sum_{n=1}^{L} I_{V L C n}=1
$$

Each user has 4 possibilities for how to connect to the hybrid system. To illustrate the equation, let's assume a 2 users case with one WiFi and one one VLC AP. Table 3.1 shows all possible options. As shown in the table, option one means that neither users is connected to neither VLC and WiFi. Thus, for the two user case, there are 16 possibilities that the proposed system runs through and selects the best case when the minimum data rate per user or the maximum average rate is maximized. However, some of these options are neglected. To be specific, the system runs through all possibilities except when the user is connected to more than one VLC AP. Algorithm 3 summarizes the proposed solution for the hybrid VLC/RF system.

Table 3.1: All posibilities for a user to connect to the proposed hybrid system

\begin{tabular}{c|cc|cc} 
& User 1 & & User 2 & \\
& $I_{R F}$ & $I_{V L C}$ & $I_{R F}$ & $I_{V L C}$ \\
\hline Option 1 & 0 & 0 & 0 & 0 \\
Option 2 & 0 & 0 & 0 & 1 \\
Option 3 & 0 & 0 & 1 & 0 \\
Option 4 & 0 & 0 & 1 & 1 \\
Option 5 & 0 & 1 & 0 & 0 \\
Option 6 & 0 & 1 & 0 & 1 \\
Option 7 & 0 & 1 & 1 & 0 \\
Option 8 & 0 & 1 & 1 & 1 \\
$\cdot$ & $\cdot$ & $\cdot$ & $\cdot$ & $\cdot$ \\
$\cdot$ & $\cdot$ & $\cdot$ & $\cdot$ & $\cdot$ \\
. & $\cdot$ & $\cdot$ & $\cdot$ & $\cdot$ \\
Option 16 & 1 & 1 & 1 & 1 \\
\hline
\end{tabular}




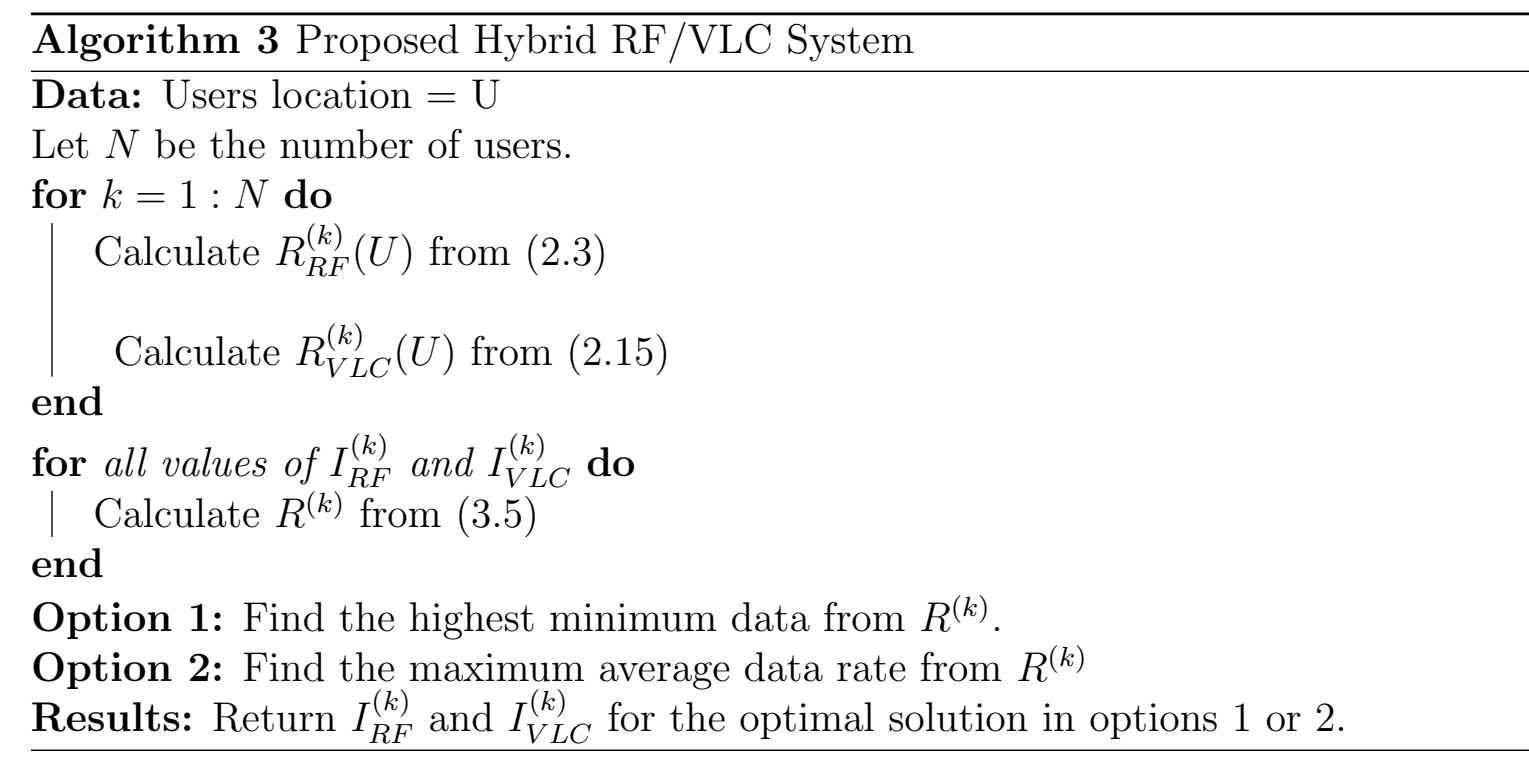




\section{Chapter 4}

\section{Numerical Results}

In this chapter, we show some results and comparisons for the proposed system. As the hybrid system consists of two independent systems, we show some results for each system that explain the range, coverage and data rate of each system. For these results, we assume a room size that can show all scenarios of the system where a user might or might not connect to a VLC AP. The room size is set to be either small (5.0 $\mathrm{m} \times 5.0 \mathrm{~m} \times 3.0 \mathrm{~m})$ or large $(8.0 \mathrm{~m} \times 8.0 \mathrm{~m} \times 3.0 \mathrm{~m})$. Two VLC AP's and only one WiFi AP are used. Figure 4.1 shows a top view of the location of the AP's in the large-room scenario simulated.

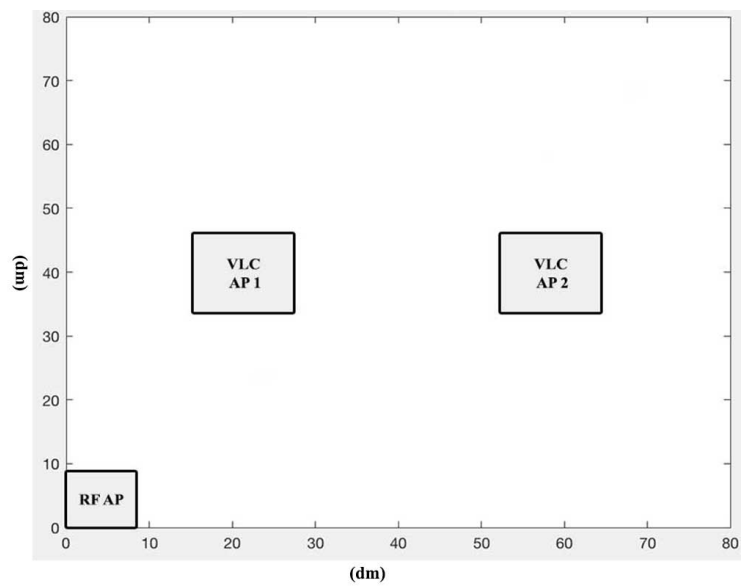

Figure 4.1: Top view on the room to show the locations of the AP's 


\subsection{WiFi Only}

In this section, we run some tests for the WiFi system to have an overall understanding of the system limits in term of coverage, data rate and received power. We assume there is no VLC connectivity. WiFi has a much wider range of coverage in comparison to the VLC system. Thus, we assume all users can connect to the WiFi AP. We also assume that all users are connected to the same channel, i.e., only one of the three channels in the $2.4 \mathrm{GHz}$ band is active. Table 4.1 shows all the parameters used in this model.

Table 4.1: Parameters used in the RF Model

\begin{tabular}{cc}
\hline$P_{t R F}$ & $100 \mathrm{~mW}$ \\
$N_{0 R F}$ & $10^{-14} \mathrm{~W} / \mathrm{Hz}$ \\
$W_{R F}$ & $20 \mathrm{MHz}$ \\
$d_{1}$ & $8 \mathrm{~m}$ \\
$\lambda$ & $0.12 \mathrm{~m}$ \\
\hline
\end{tabular}

The maximum data rate we can achieve using this model for a single user is 120 Mbps using (2.3). With increased range, we notice a significant drop to about $20 \mathrm{Mbps}$ for users located at 8 meters from the WiFi AP. Figure 4.2 shows the relationship between data rate and distance in the WiFi system.

Another issue we should consider is the relationship between the transmitted power and throughput for a user. RF standards allow the RF AP to transmit up to $100 \mathrm{~mW}$, which is the maximum allowed transmitted power for WiFi AP. Thus, we tested the effect of transmitting power from $1 \mathrm{~mW}$ to $100 \mathrm{~mW}$ on the data rate for a user located at a $1 \mathrm{~m}$ distance from the WiFi AP. Figure 4.3 shows the results using (2.3). As expected, the higher the transmitted power, the better the throughput we can achieve. 


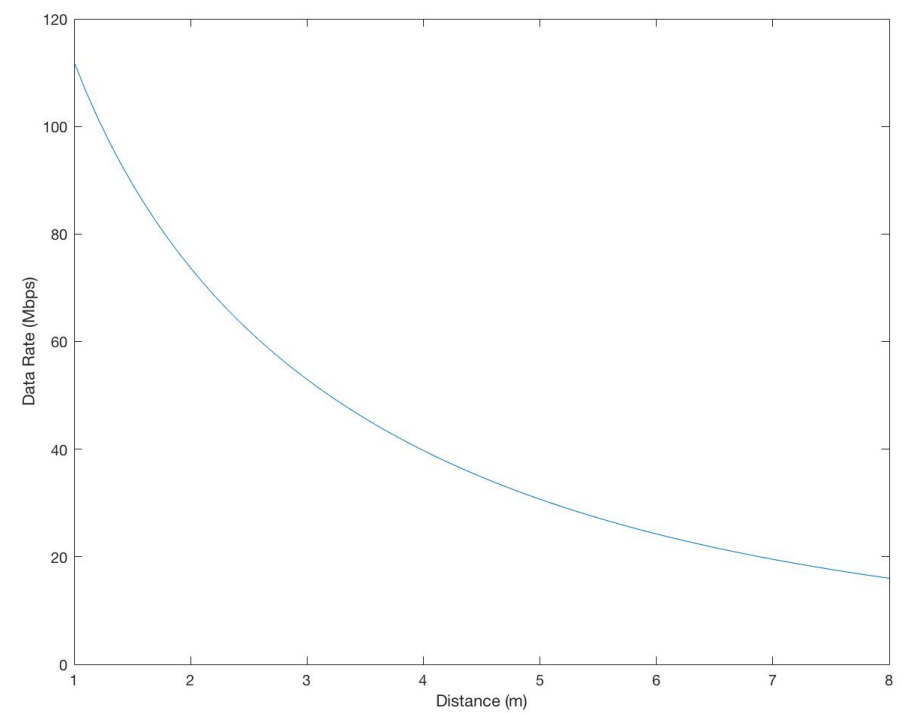

Figure 4.2: The relationship between data rate and distance for a WiFi system using the parameters in Table 4.1

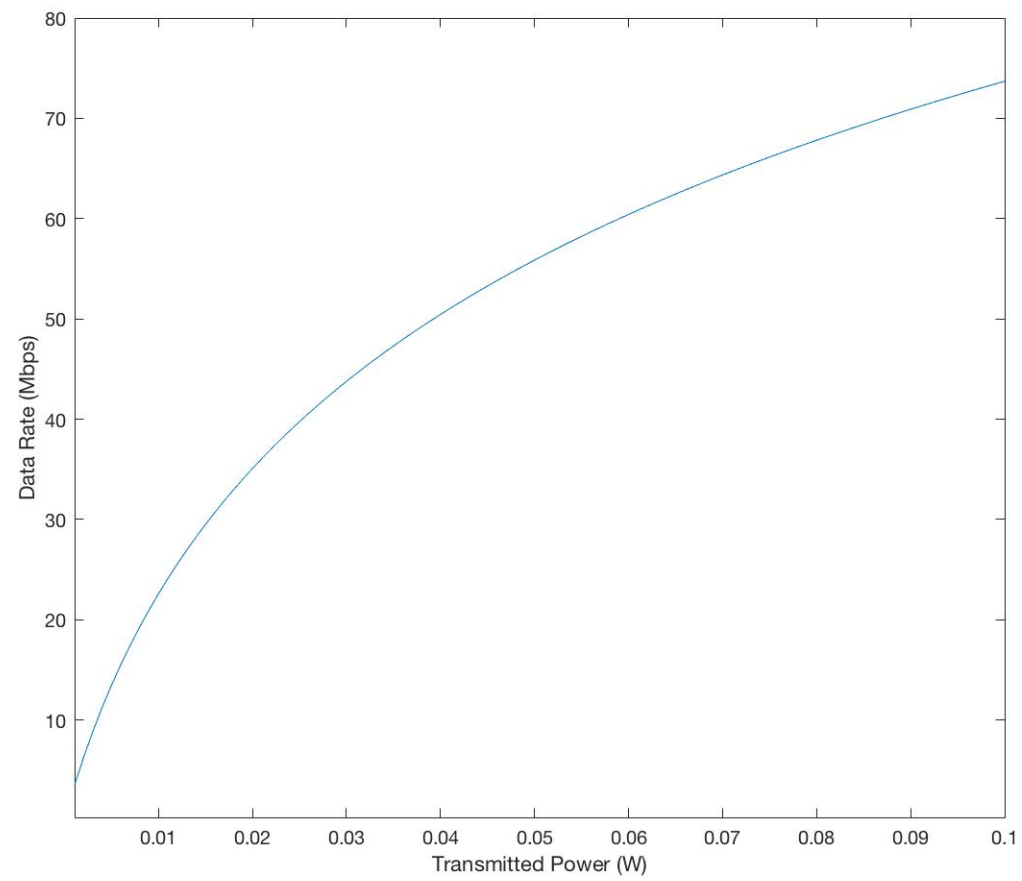

Figure 4.3: The relationship between the data rate and transmitted power in RF system assuming the parameters in Table 4.1. for a user $1 \mathrm{~m}$ away from the AP. 


\subsection{VLC}

In this section, we assume there is no WiFi connectivity to test the characteristics of the VLC system. We use the VLC model in Section 2.3 with the 25-LED lamp. For the light source, we assume each LED transmits $0.4 \mathrm{~W}$, which means a total of $10 \mathrm{~W}$ from each VLC AP power is transmitted. We use a semi-angle of 8 degree at half power for each LED. Each receiver is assumed to have a photodetector with responsivity of $0.3 \mathrm{~A} / \mathrm{W}$. The minimum SINR is calculated by setting the maximum acceptable BER to $10^{-3}$ in (2.13) with constellation modulation size $M_{k}=2$. Using (2.13), the minimum SINR to be connected to any AP's is 13.36 dB. Similar to WiFi, the channel bandwidth is set to be $20 \mathrm{MHz}$, and the noise spectral density is assumed to be $10^{-18} \mathrm{~W} / \mathrm{Hz}[26]$. The table below summarizes the parameters used in the VLC system.

Table 4.2: Parameters used in the VLC Model

\begin{tabular}{cc}
\hline Large room size & $8 \mathrm{~m} \times 8 \mathrm{~m} \times 3 \mathrm{~m}$ \\
$V L C_{A P 1}$ location & $(2,4,3) \mathrm{m}$ \\
$V L C_{A P 2}$ location & $(6,4,3) \mathrm{m}$ \\
\hline Small room size & $5 \mathrm{~m} \times 5 \mathrm{~m} \times 3 \mathrm{~m}$ \\
$V L C_{A P 1}$ location & $(1.25,2.5,3) \mathrm{m}$ \\
$V L C_{A P 2}$ location & $(3.75,2.5,3) \mathrm{m}$ \\
\hline$P_{t V L C}$ & $10 \mathrm{~W}$ \\
Semi-angle & $8^{\circ}$ \\
$S N R_{\min }$ & $13.36 \mathrm{~dB}$ \\
$B E R_{\min }$ & $10^{-3}$ \\
$N_{0 V L C}$ & $10^{-18} \mathrm{~W} / \mathrm{Hz}[26]$ \\
$W_{V L C}$ & $20 \mathrm{MHz}$ \\
Responsivity & $0.3 \mathrm{~A} / \mathrm{W}$ \\
\hline
\end{tabular}

In Figure 4.4, we illustrate the power distribution on the floor from both AP's. We notice that the received power near the AP's is much higher than in the other 
parts of the room, which indicates that users in areas with weak coverage from the VLC may not be connected, as their $S I N R$ may be lower than the minimum $S I N R$.

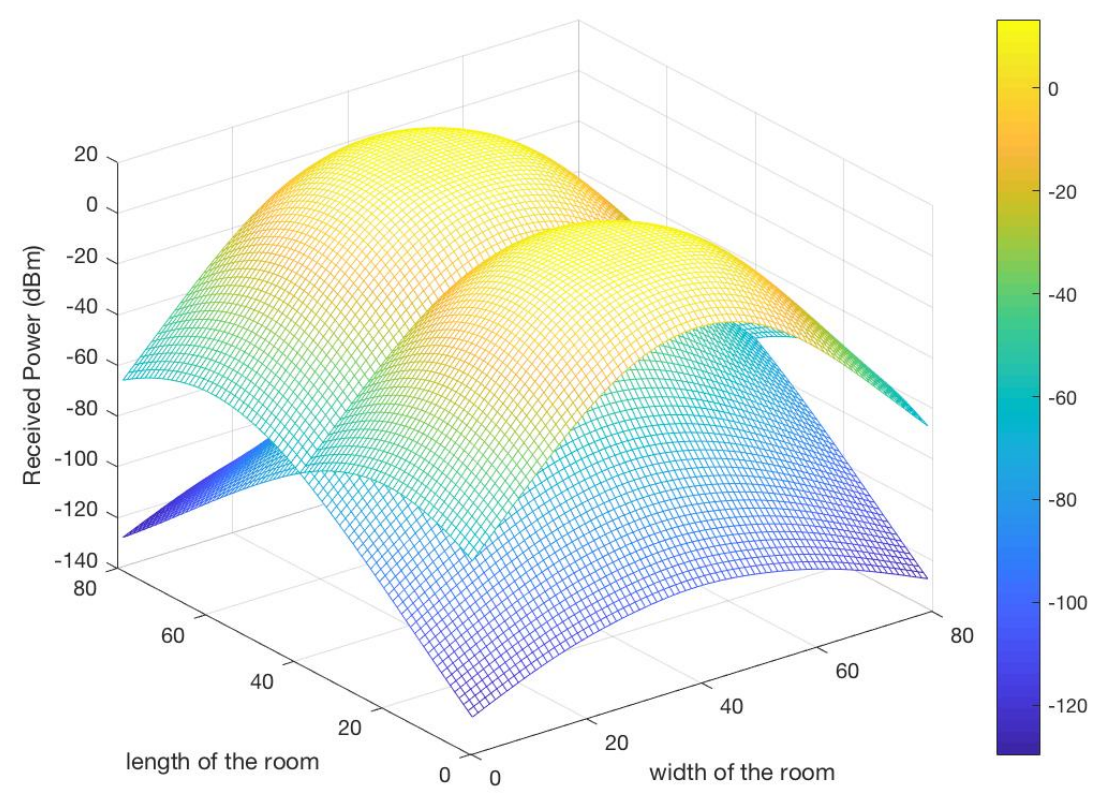

Figure 4.4: Received power distribution on the floor for the assumptions in Table 4.2

Figure 4.5 shows the effects of the transmitted power on the SNR for a single user. By varying the peak power for one LED from 0 to $2 \mathrm{~W}$, the transmitted power from one lamp varies from 0 to $50 \mathrm{~W}$. The receiver location is fixed on the floor directly under the VLC AP to test the effects. We notice that the transmitted power significantly affects the SNR.

\subsection{Hybrid VLC and WiFi}

In this section, we show numerical results of the proposed hybrid system for each of the following options:

- a fair option where the highest minimum data rate is found. 


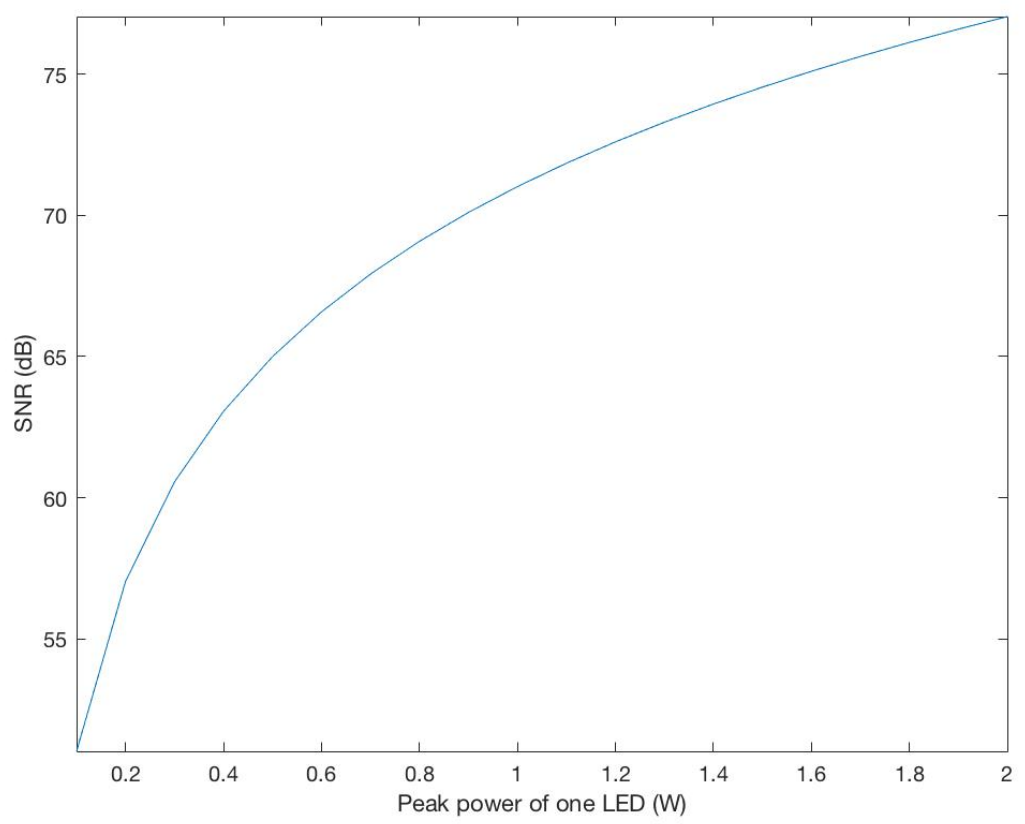

Figure 4.5: The relationship between the peak power of the LED and the SNR assuming the receiver is $3 \mathrm{~m}$ directly below the lamp.

- a highest throughput option where the maximum total data rate is found.

For each option, we compare the following models:

- Model (1): The optimized solution from the proposed system.

- Model (2): Connect to the best link (VLC or WiFi).

- Model (3): Always connect to both VLC and WiFi.

- Model (4): WiFi only.

Note that we cannot compare the results to the stand-alone VLC system as some users might not connect due to the coverage of the VLC, which results in an unfair comparison with the other models. In model (2), the user can transmit data using only one of the systems. The user connects automatically to the link with the highest 
data rate. The data rate in this model can be calculated as

$$
R^{(k)}=\max _{I_{R F}, I_{V L C}}\left(\frac{\left(R_{R F}^{(k)} * I_{R F}^{(k)}\right)}{\sum_{1}^{N} I_{R F}^{(n)}},\left(\frac{\left(R_{V L C 1}^{(k)} * I_{V L C 1}^{(k)}\right)}{\sum_{1}^{N} I_{V L C 1}^{(n)}}+\ldots+\frac{\left(R_{V L C L}^{(k)} * I_{V L C L}^{(k)}\right)}{\sum_{1}^{N} I_{V L C L}^{(n)}}\right)\right)
$$

In model (3), the user is always receiving data on any system it can connect to. If the user can connect to more than one VLC AP, it receives only on the AP with the highest data rate. In this model, the data rate is always calculated using the following equation:

$$
R^{(k)}=\left(\frac{\left(R_{R F}^{(k)} * I_{R F}^{(k)}\right)}{\sum_{1}^{N} I_{R F}^{(n)}}+\left(\frac{\left(R_{V L C 1}^{(k)} * I_{V L C 1}^{(k)}\right)}{\sum_{1}^{N} I_{V L C 1}^{(n)}}+\ldots+\frac{\left(R_{V L C L}^{(k)} * I_{V L C L}^{(k)}\right)}{\sum_{1}^{N} I_{V L C L}^{(n)}}\right)\right)
$$

To test the effectiveness of the proposed system, we simulate the models over two cases:

- a large room size $(8 \mathrm{~m} \times 8 \mathrm{~m} \times 3 \mathrm{~m})$ where the users do not have fair access to both VLC and WiFi as the size of the room is large.

- a small room size $(5 \mathrm{~m} \times 5 \mathrm{~m} \times 3 \mathrm{~m})$ where the users have fair access to both VLC and WiFi.

For each case, we run the optimization using both SNR and SINR in the VLC system. The SINR constraint is appropriate when the VLC-AP signals interfere with one another. Conversely, the SNR constraint assumes a method is used to eliminate the co-channel interference (outside the scope of this thesis). The goal is to understand if it is worth working to remove the adjacent channel interference or not. One possible solution to avoid the co-channel interference might be by having each VLC-AP modulate a color that is different from the color of the adjacent VLC-AP's. In this way, the AP's cannot transmit with the same color in the same time to avoid the co-channel interference. Another approach might be to restrict the receiver FOV to 
avoid interference.

\subsubsection{Optimal Fair Allocation}

In this section, we compute the largest minimum data rate that we can achieve per user in the different systems and compare it to the optimized solution in the proposed system. In each of the following studies, the users are distributed uniformly in the room, and the results are averaged over 50 trials. The room size is $(8 \mathrm{~m} \times 8 \mathrm{~m} \times 3$ m). Figure 4.6 shows that the VLC does not cover all the locations in the room.

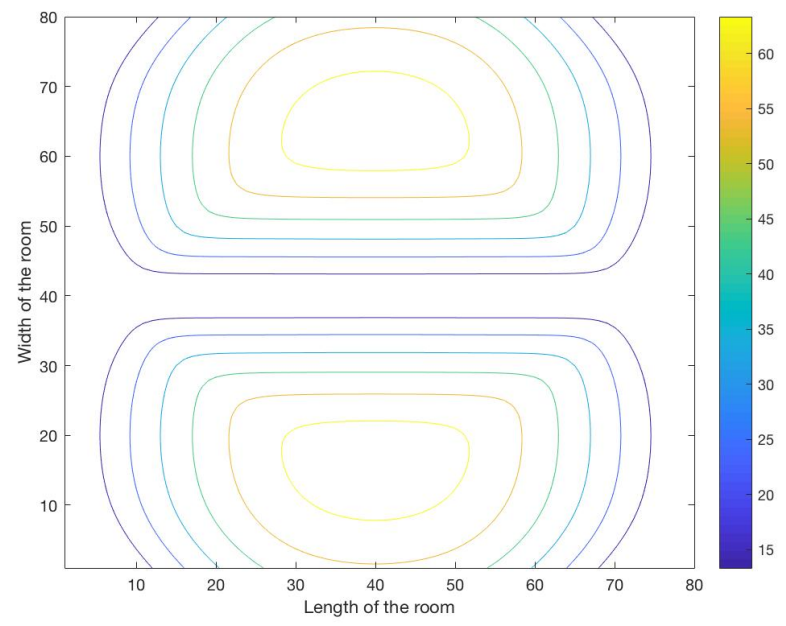

Figure 4.6: SINR coverage of VLC in a room of size $(8 \mathrm{~m} \times 8 \mathrm{~m} \times 3 \mathrm{~m})$

To begin, we compare the optimized solution of the proposed system with the WiFi system to illustrate the effects of using a hybrid system in RF environments. Figure 4.7 shows this comparison. When the VLC AP's do not experience co-channel interference and 2 users are connected, the minimum data rate for a user in the optimized solution is improved by approximately 8 times compared to the WiFi-only system when we use the proposed system. As the number of users increases, we notice a slight increase in the improvements. For example, in the case of 7 users, the minimum data rate for a user in the proposed system and the WiFi are $16.58 \mathrm{Mbps}$ 
and 1.69 Mbps, respectively. This indicates that the proposed system can improve the minimum data rate by approximately 10 times compared to the WiFi stand-alone system. When we consider the co-channel interference between the VLC AP's, the minimum data rate for the proposed system drops by 18.6-32.6\%, depending on the number of users.

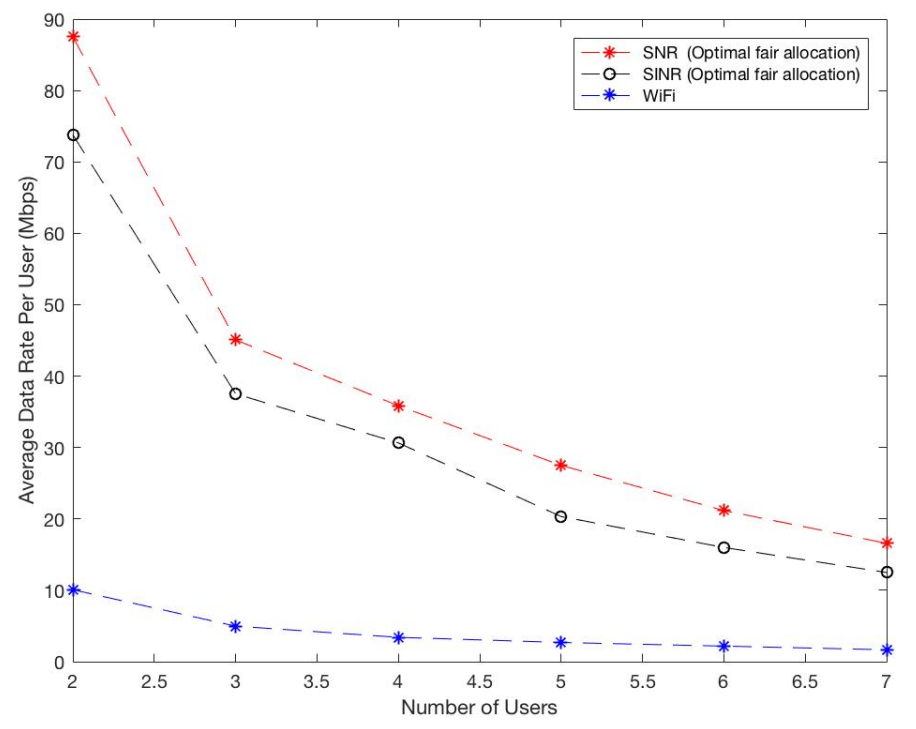

Figure 4.7: Comparison between $\mathrm{WiFi}$ and the proposed hybrid system in term of minimum data rate for parameters listed in Tables 4.1 and 4.2.

The next step is to compare the optimized solution with the other hybrid systems. In Figure 4.8, we compare the minimum data rate per user for the proposed system with the other hybrid systems assuming the VLC AP's do not experience co-channel interference. The results show that the proposed system is always better than the other two hybrid systems in terms of the minimum data rate per user. When 2 users are connected, the proposed system has $10.9 \%$ and $4.5 \%$ higher minimum data rate compared to the other two models. As the number of users increases, the relative difference among these hybrid models increases. For example, when 7 users are connected, the minimum data rate per user for the optimized solution is 16.6 Mbps 
while the other two models have 12.86 Mbps and 6.5 Mbps. This shows that the proposed system has $29 \%$ and $155 \%$ higher minimum data rate than the other two models. When the VLC AP's experience co-channel interference, Figure 4.9 shows the results. We notice that the performance of all hybrid systems are affected by the co-channel interference, but the difference between them remains almost the same, as the proposed system always has the best performance.

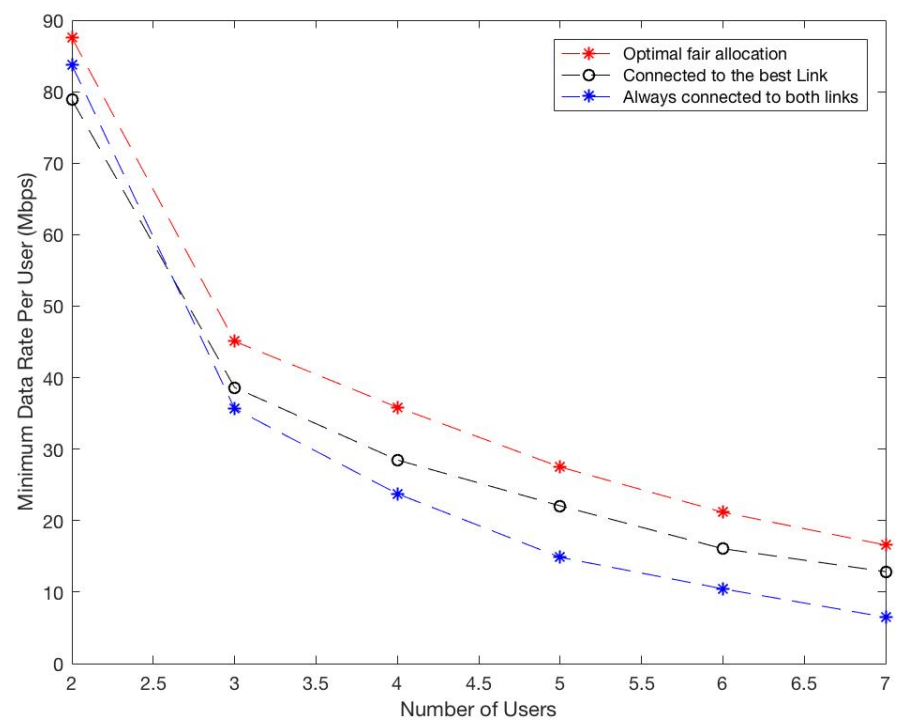

Figure 4.8: Comparison between the hybrid systems in term of the minimum data rate for parameters listed in Tables 4.1 and 4.2 assuming the VLC AP's do not experience co-channel interference

The following simulation is computed in a room of size $(5 \mathrm{~m} \times 5 \mathrm{~m} \times 3 \mathrm{~m})$ to let all users have a fair access to both VLC and WiFi. The users are distributed uniformly in the room, and the results are averaged over 50 trials. Figure 4.10 shows the coverage of the VLC when we consider the co-channel interference between the VLC AP's. 


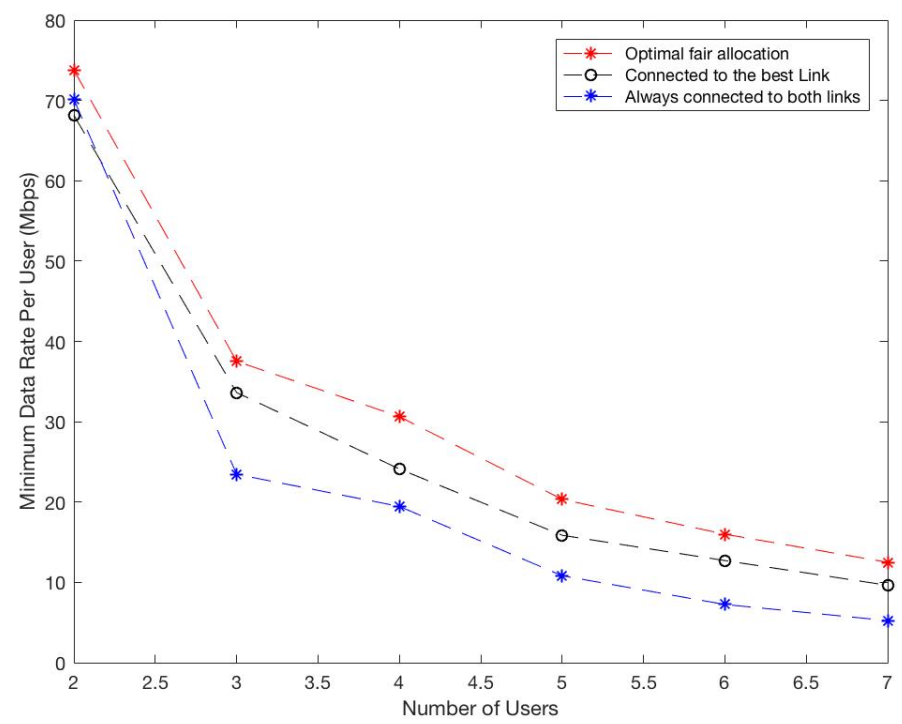

Figure 4.9: Comparison between the hybrid systems in term of the minimum data rate when the VLC AP's experience co-channel interference for parameters listed in Tables 4.1 and 4.2 .

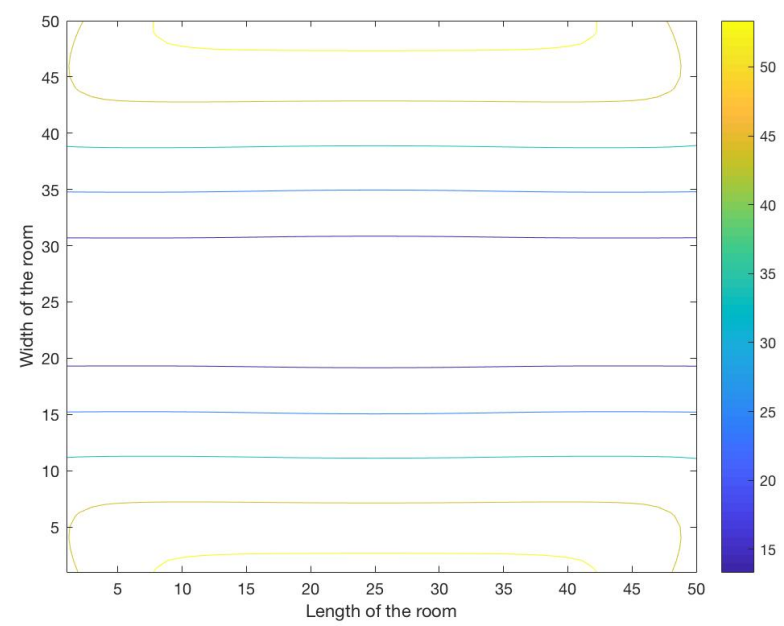

Figure 4.10: SINR coverage of VLC in a room of size $(5 \mathrm{~m} \times 5 \mathrm{~m} \times 3 \mathrm{~m})$

In Figure 4.11, we compare all the models when we consider the co-channel interference between the VLC AP's. As shown in the figure, the proposed system achieves the highest minimum data rate among all models. When two users are connected, the 
simulation shows that the optimized minimum data rate per user in the proposed system is 3.5 times higher than the WiFi stand alone system. In comparison to the other two models, the proposed system has $17 \%$ and $3 \%$ higher minimum data rate. As the number of users increases, the minimum data rate per user improvement increases. When 7 users are connected, the optimized solution is 4.6 times higher than the WiFi stand alone system. In comparison to the other two models, the optimized solution has $19 \%$ and $151 \%$ higher minimum data rate per user. When we assume there is no co-channel interference between the VLC APs, Figure 4.12 shows the results of this comparisons. As shown in the figure, the difference between the hybrid systems remains the same but the overall performance of the hybrid systems compared with the stand-alone WiFi system increases significantly. For example, when 6 users are connected, the minimum data rate for the proposed system is $66.3 \mathrm{Mbps}$, while the minimum data rate for the $\mathrm{WiFi}$ system is $4.5 \mathrm{Mbps}$. This indicate that the proposed system has a 14 times improvement over the WiFi system compared to the 5.6 times improvement when we consider the co-channel interference.

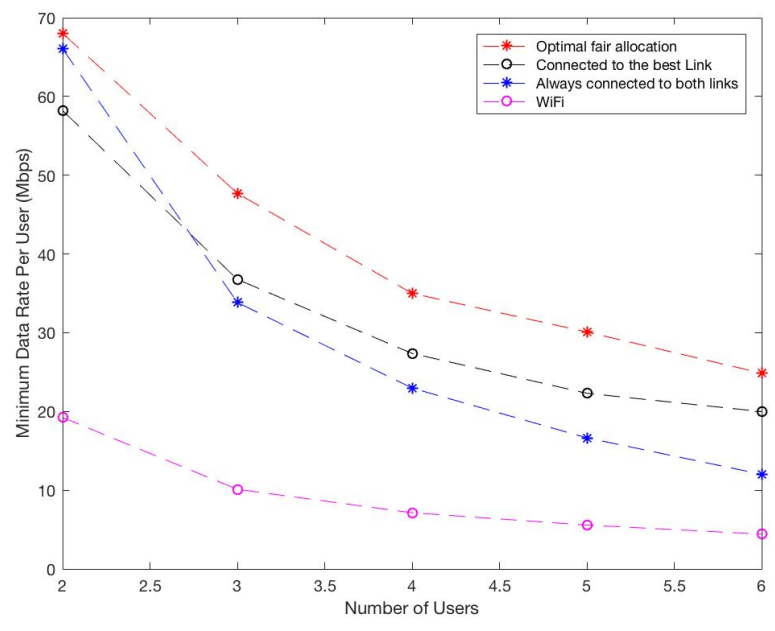

Figure 4.11: Comparison between the hybrid systems in term of the minimum data rate in the small room when the VLC AP's experience co-channel interference for parameters listed in Tables 4.1 and 4.2 . 


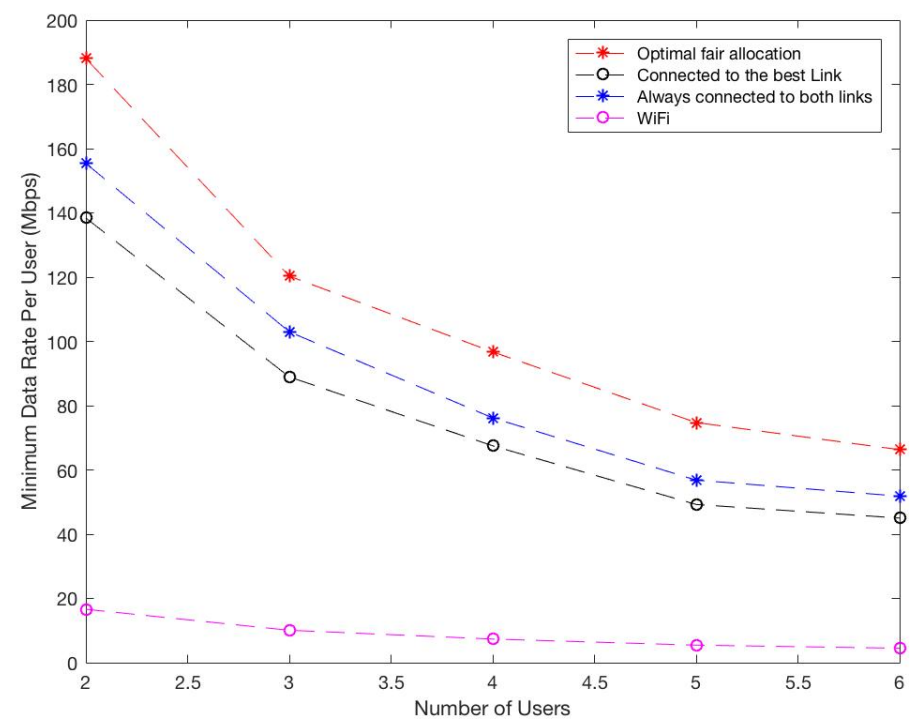

Figure 4.12: Comparison between the hybrid systems in term of the minimum data rate in the small room when the VLC AP's do not experience co-channel interference for parameters listed in Tables 4.1 and 4.2.

\subsubsection{Maximum Throughput Allocation}

In this section, we compute the maximum average data rate that we can achieve per user in the different systems and compare it to the optimized solution in the proposed system. In each of the following studies, the users are distributed uniformly in the room, and the results are averaged over 50 trials. The large room size is $(8 \mathrm{~m}$ $\times 8 \mathrm{~m} \times 3 \mathrm{~m})$, and the small room size is $(5 \mathrm{~m} \times 5 \mathrm{~m} \times 3 \mathrm{~m})$.

To begin, we compare the proposed hybrid system with the stand-alone WiFi system with and without considering the co-channel interference between the VLC AP's. Figure 4.13 shows a comparison of the average data rate between the two options in the proposed system and the WiFi assuming there is co-channel interference. As shown in the figure, both options in the proposed system have substantially higher average data rate than the $\mathrm{WiFi}$ system. As the number of users increases, the relative differences among these systems increases. Overall, the maximum throughput 
allocation has 6-11 times higher average data rate than the stand-alone WiFi system. The average data rate in the optimal fair allocation is also higher than the stand-alone WiFi system, by approximately 6-8 times based on the number of users.

When we assume there is no co-channel interference between the VLC-AP's, the performance of the proposed system improves, as shown in Figure 4.14. The maximum throughput allocation now has 8-13 times higher average data rate than the standalone WiFi system based on the number of users. We notice that the co-channel interference between the VLC AP's does not affect the results as much as it affects the minimum data rate per user, as the performance of the maximum throughput allocation drops by (16-7)\% when we consider the co-channel interference while the performance of the optimal fair allocation drops by (18-32)\%.

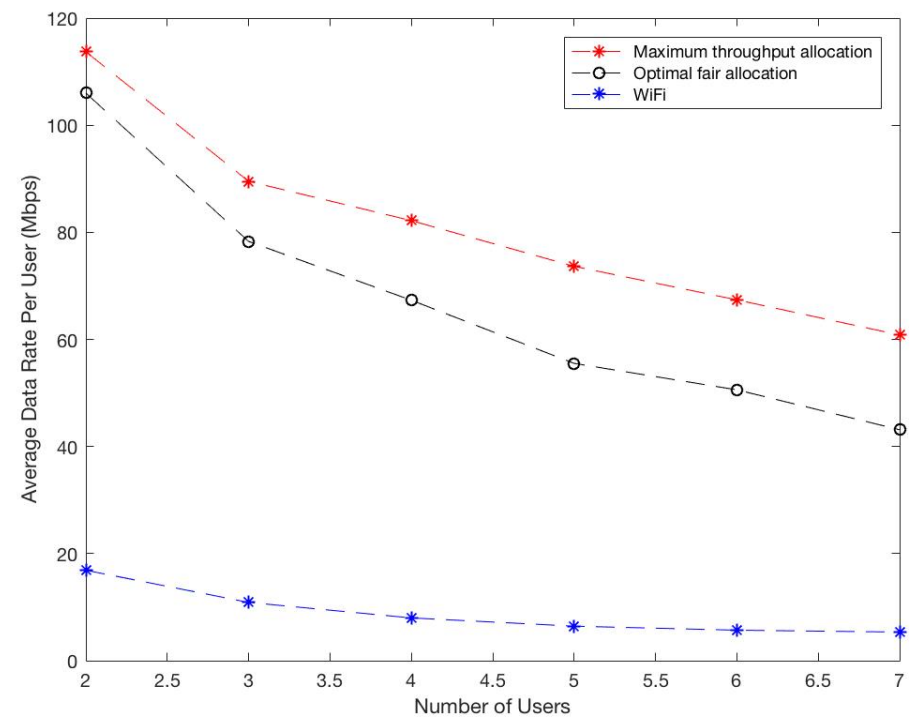

Figure 4.13: Comparison between the proposed system and WiFi in term of the average data rate when the VLC AP's experience co-channel interference for parameters listed in Tables 4.1 and 4.2. 


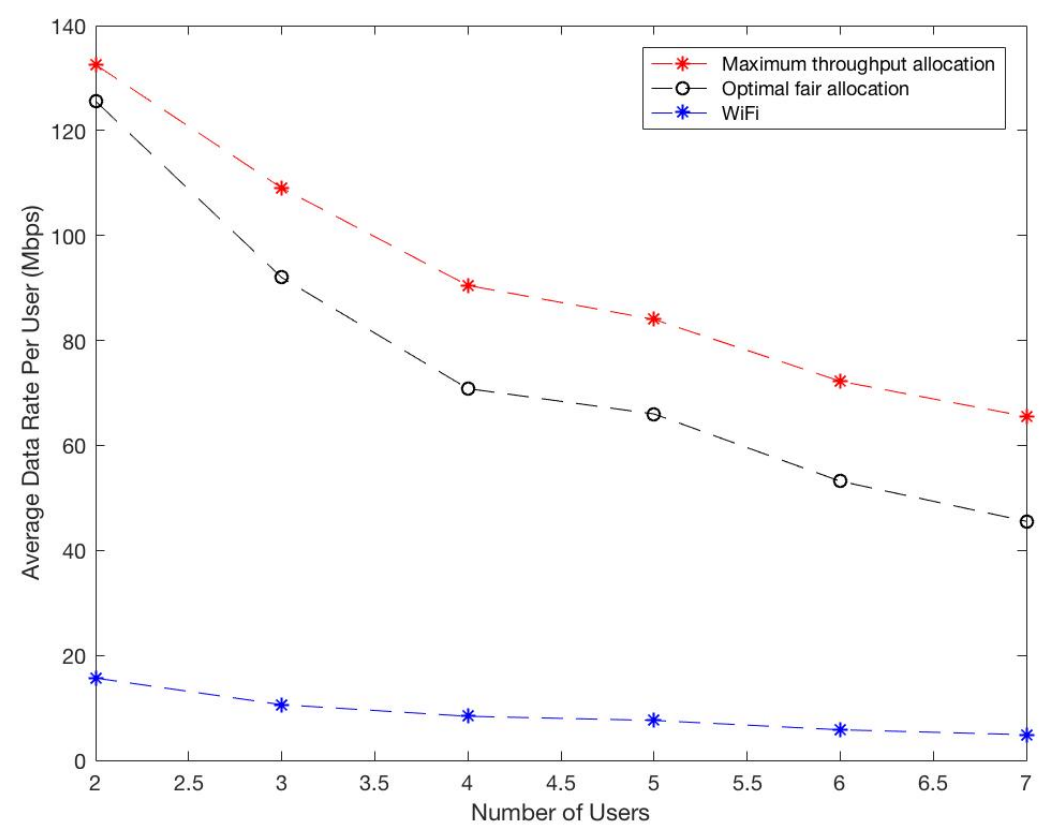

Figure 4.14: Comparison between the proposed system and $\mathrm{WiFi}$ in term of the average data rate when the VLC AP's do not experience co-channel interference for parameters listed in Tables 4.1 and 4.2.

The next step is to compare the average data rate over all hybrid systems. The simulation shows that all hybrid systems have almost the same performance in term of the average data rate. Figure 4.15 shows the results of the simulation when we consider the co-channel interference between the VLC AP's. As shown in the figure, the maximum throughput allocation achieves the highest average data rate for both low and high user densities. All fair hybrid systems, including the proposed system when optimizing fair allocation, show a similar performance in term of the average data rate. When we assume there is no co-channel interference between the VLC-AP's, the performance of all hybrid systems improve as shown in Figure 4.16. However, the gap between the maximum throughput allocation and the other models remains almost the same. 


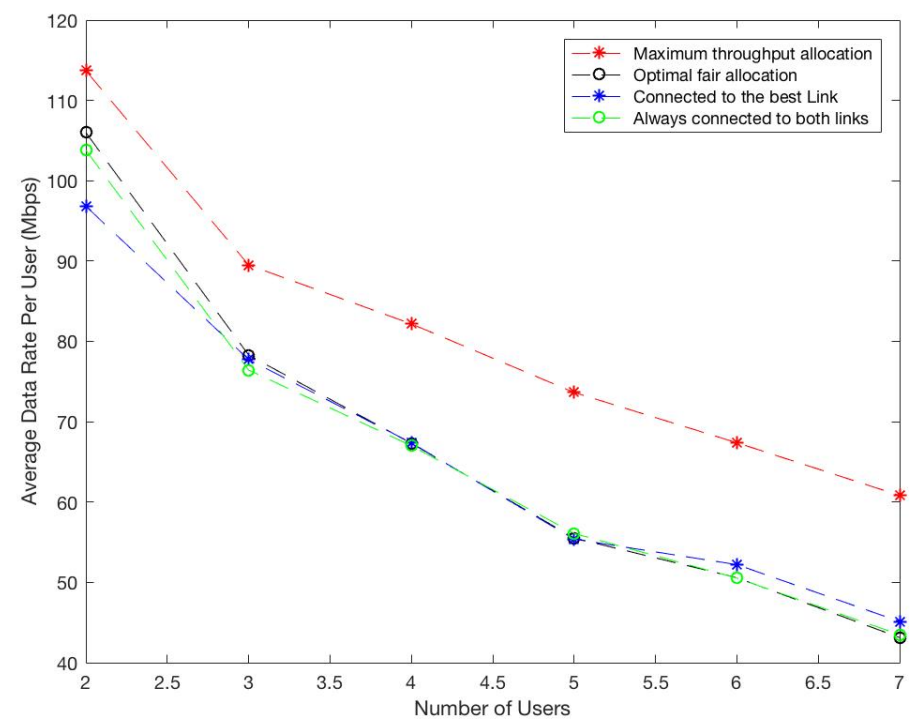

Figure 4.15: Comparison between all the systems in term of the average data rate when the VLC AP's experience co-channel interference for parameters listed in Tables 4.1 and 4.2 .

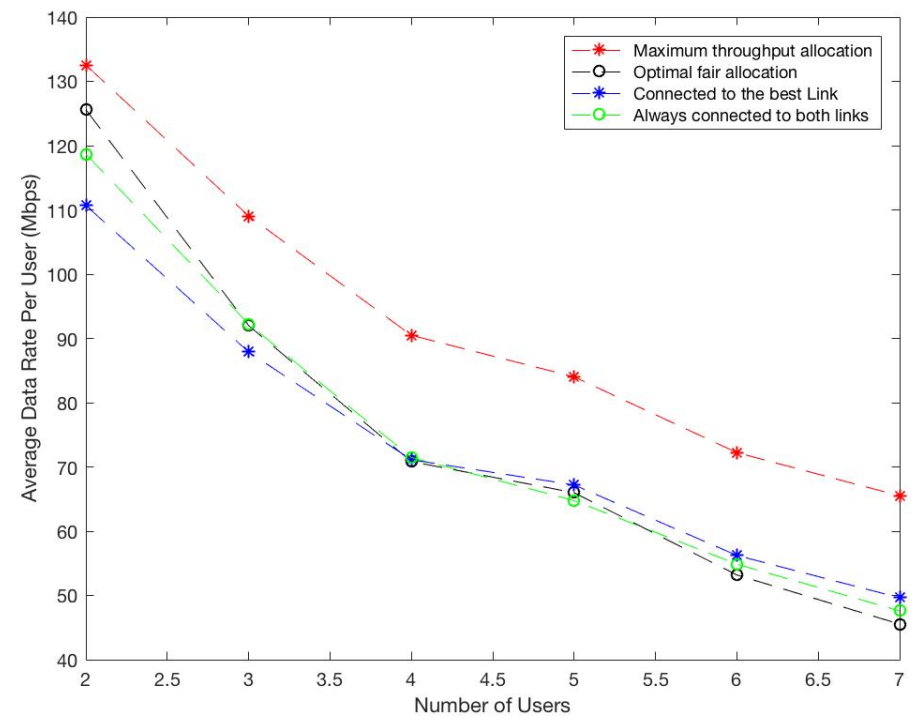

Figure 4.16: Comparison between all the systems in term of the average data rate when the VLC AP's do not experience co-channel interference for parameters listed in Tables 4.1 and 4.2 . 
The following simulation is computed in a small room of size $(5 \mathrm{~m} \times 5 \mathrm{~m} \times 3$ m) for all models with and without considering the co-channel interference. Figure 4.17 shows the simulation for all the models when we consider the co-channel interference. There is no significant difference from the previous results as the maximum throughput allocation achieves the highest average data rate per user among all systems. We notice that the average data rate in all systems increases compared to the larger room size since all users have better coverage from both links. For example, when 6 users are connected, the average data rate for models (1), (2), (3), and (4) are 61.65 Mbps, 47.7 Mbps, 47.16 Mbps, and 7.71 Mbps, respectively. This indicates that the proposed model is better than the other models by $29.2 \%, 30.7 \%$, and $700 \%$, respectively.

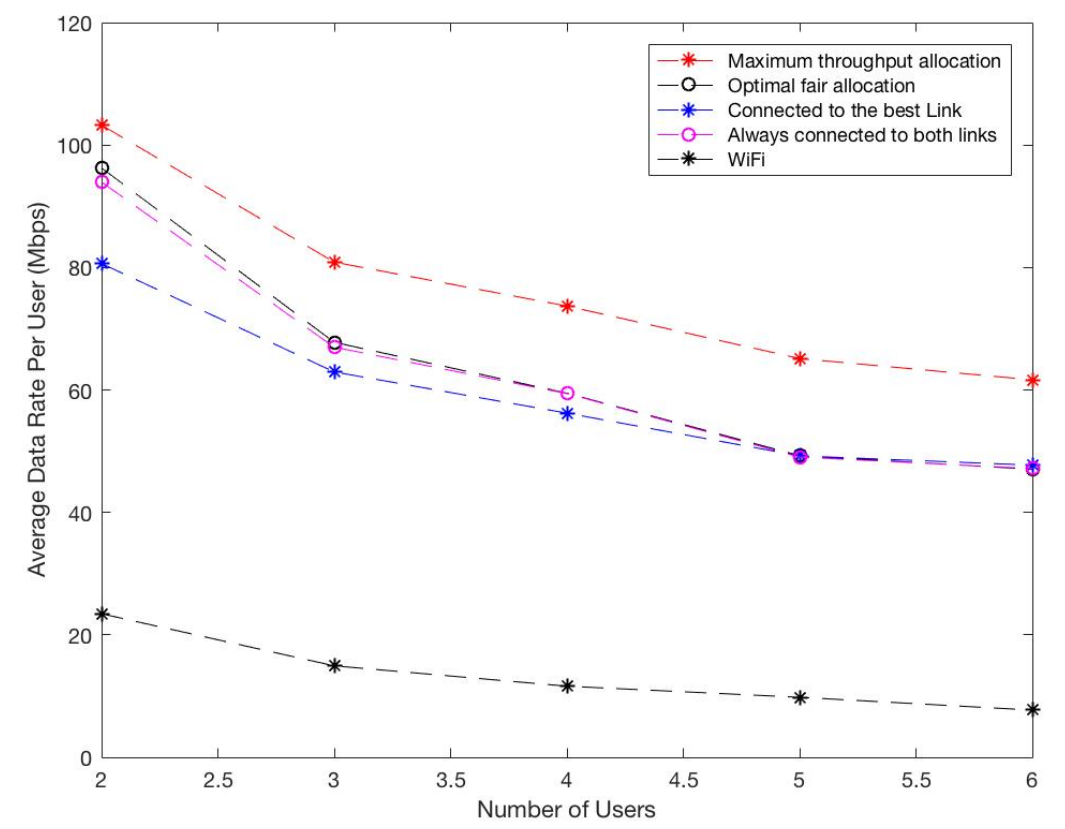

Figure 4.17: Comparison between all the systems in term of the average data rate for the small room when the VLC AP's experience co-channel interference for parameters listed in Tables 4.1 and 4.2 . 
When we assume there is no co-channel interference between the VLC AP's, the performance of the hybrid systems improves as shown in Figure 4.18. For example, when 6 users are connected, the average data rate for models (1), (2), (3), and (4) are 89.2 Mbps, 68.2 Mbps, 75.5 Mbps, 8.0 Mbps, respectively. This indicates that the performance of the hybrid models improved by $30.6 \%, 18 \%, 1013 \%$, respectively. As expected, the co-channel interference affects all hybrid systems about the same, as there is no significant difference between the improvement in the performance of all hybrid systems.

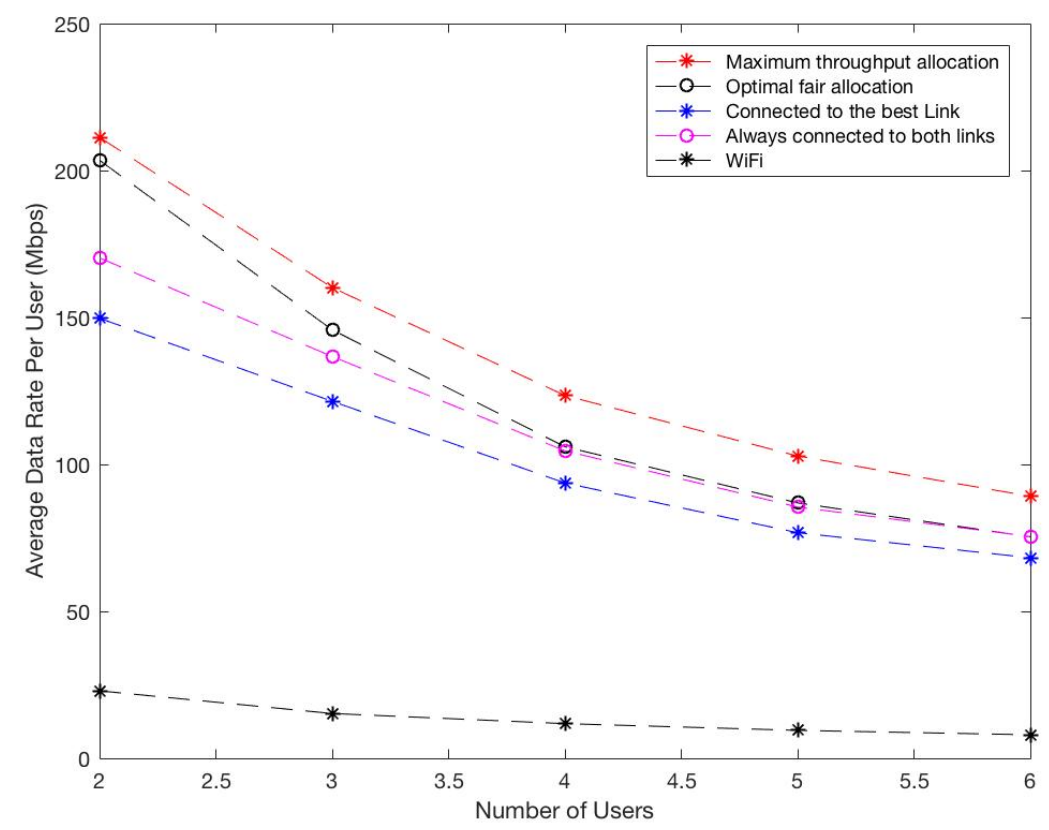

Figure 4.18: Comparison between all the systems in term of the average data rate for the small room when the VLC AP's do not experience co-channel interference for parameters listed in Tables 4.1 and 4.2 .

One significant disadvantage of optimizing the maximum throughput allocation is that the system might disconnect some users to achieve the highest possible average data rate. For a low user density environment, the maximum throughput allocation selects the best case to achieve the highest average data rate. However, once 
the number of users exceeds $L$, the number of AP's (3 in this case), the maximum throughput allocation selects only the $L$ users with the highest data rate in each link and disconnects the others. On the other hand, the simulations show that the optimal fair allocation shows a significant improvement in maximizing the minimum data rate without penalty to the average data rate. Thus, performing similar results in term of the average data rate while achieving the highest possible minimum data rate is a significant advantage for the proposed fair system.

\subsubsection{Case Study}

In this section, we illustrate two cases from the simulation using the SNR (zero interference). The goal of this section is to understand how each system affects the average and minimum data rates. The first case selected tests a low user density environment with only three users. The case is selected so that one user can access only the WiFi while the other two users can access both the WiFi and VLC. The room size is $(8 \mathrm{~m} \times 8 \mathrm{~m} \times 3 \mathrm{~m})$ as shown in Figure 4.1 and there are only two VLC-AP's. In this way, we can test the proposed system when the users do not have fair access to both links. The location of users 1, 2, and 3 are $[7.8 \mathrm{~m}, 4.6 \mathrm{~m}, 1 \mathrm{~m}],[2.2 \mathrm{~m}, 1.1 \mathrm{~m}$, $1 \mathrm{~m}],[1.3 \mathrm{~m}, 2.2 \mathrm{~m}, 1 \mathrm{~m}]$, respectively. When applying these locations in the WiFi and VLC systems, the maximum data rates for the WiFi system are calculated to be 11.9 Mbps, 94.5 Mbps, and 91.3 Mbps for the three users, respectively. For the VLC system, only two users can connect to the VLC-AP. User 1 can connect to VLC-AP2 with $60 \mathrm{Mbps}$, and user 3 can connect to VLC-AP1 also with 60 Mbps. User 2 cannot connect to any VLC-AP. Table 4.3 summarizes the results from running the code.

When implementing these results in the proposed system, the solution connects user 1 to the VLC-AP2, connects user 2 to the WiFi-AP, and connects user 3 to the VLC-AP1. The minimum data rate we achieve is $60 \mathrm{Mbps}$, while the average data 
Table 4.3: The maximum data rate based on distance for case 1

\begin{tabular}{cccc} 
& User 1 & User 2 & User 3 \\
\hline$R_{R F}$ & $11.9 \mathrm{Mbps}$ & $94.5 \mathrm{Mbps}$ & $91.3 \mathrm{Mbps}$ \\
$R_{V L C-A P 1}$ & 0 & 0 & $60 \mathrm{Mbps}$ \\
$R_{V L C-A P 2}$ & $60 \mathrm{Mbps}$ & 0 & 0 \\
\hline
\end{tabular}

rate is $71.5 \mathrm{Mbps}$. In the second hybrid system where the user only connects to one of the two links, the solution connects user 1 to the VLC-AP2, connects user 2 to the $\mathrm{WiFi}-\mathrm{AP}$ and connects user 3 to the $\mathrm{WiFi}-\mathrm{AP}$, as the data rate is higher than the data rate from VLC-AP1. In this case, the minimum data rate is calculated to be 45.7 Mbps while the average data rate is $51 \mathrm{Mbps}$. We notice that VLC-AP2 is not used even though user 3 can connect to it. At the same time, the WiFi capacity is shared among these two users.

In the third hybrid system where the user always connects to both systems, the solution tends to let user 1 connect to WiFi-AP and VLC-AP2, user 2 connect to WiFi-AP and user 3 connect to WiFi-AP and VLC-AP1. The minimum data rate is calculated to be $31.5 \mathrm{Mbps}$ while the average data rate is $62 \mathrm{Mbps}$. In this case, the WiFi channel capacity is divided among all users even though user 3 does not need to connect to the WiFi as it has a high data rate from VLC-AP1. Table 4.4 summarizes the results for the three hybrid systems. The table shows that the proposed system improves the channel capacity and allows the users to achieve the best results.

Table 4.4: Comparison between the hybrid systems in term of minimum and average data rate for 3 users.

Optimized min rate Optimized average rate

\begin{tabular}{lcc}
\hline Model (1): Proposed system & $60 \mathrm{Mbps}$ & $71.5 \mathrm{Mbps}$ \\
Model (2): Connected to the best system & $31.5 \mathrm{Mbps}$ & $62 \mathrm{Mbps}$ \\
Model (3): Connected to both systems & $45.7 \mathrm{Mbps}$ & $51 \mathrm{Mbps}$ \\
\hline
\end{tabular}


Let's assume another case with a dense user environment. In this case, we randomly select a case with 7 users. Table 4.5 shows the resulting data rate based on distance for all users from each AP. All users can access at least one VLC AP and the WiFi.

Table 4.5: Calculated data rate in Mbps for all users from each AP's

\begin{tabular}{cccccccc} 
& User 1 & User 2 & User 3 & User 4 & User 5 & User 6 & User 7 \\
\hline$R_{R F}$ & 47.54 & 40.82 & 27.25 & 123.75 & 21.32 & 54.48 & 49.49 \\
$R_{V L C 1}$ & 40 & 60 & 0 & 60 & 0 & 100 & 40 \\
$R_{V L C 2}$ & 80 & 0 & 80 & 0 & 60 & 0 & 0 \\
\hline
\end{tabular}

Table 4.6 presents the results of implementing the proposed hybrid system. When the indicator equals 1 , this means the user is connected to the AP, and similarly, when the indicator equals 0 , it means the user is not connected to the AP. When we implement the optimal fair allocation in the proposed hybrid system, 4 users connect to the WiFi, 3 users connect to VLC-AP1 while 3 users connect to VLC-AP2. The proposed system allows users 2 and 7 to connect to both the WiFi and VLC-AP1 to achieve the highest possible minimum data rate.

Table 4.6: Indicator variable for each user when implementing the optimal fair allocation (Model (1)).

\begin{tabular}{c|ccccccc} 
& User 1 & User 2 & User 3 & User 4 & User 5 & User 6 & User 7 \\
\hline$I_{R F}$ & 0 & 1 & 0 & 1 & 1 & 0 & 1 \\
$I_{V L C 1}$ & 0 & 1 & 0 & 0 & 0 & 1 & 1 \\
$I_{V L C 2}$ & 1 & 0 & 1 & 0 & 1 & 0 & 0 \\
\hline$R$ (Mbps) & 26.7 & 30.2 & 26.7 & 30.94 & 25.33 & 33.3 & 25.7
\end{tabular}

Table 4.7 presents the output of implementing the hybrid system where the user always connect to both the RF and VLC systems. We notice that the WiFi channel capacity is divided among all users, regardless of their data rate or whether or not 
they can connect to VLC. VLC-AP1 is also divided among 4 out of the 7 users while VLC-AP2 is only shared among three users. In term of the effect on the WiFi capacity, having a hybrid system does not solve the problem, as no matter how many AP's are available, the user will always connect to the WiFi. The minimum data rates for the optimal fair allocation and the hybrid system, where the users always connect to both links, are 25.44 Mbps and $17 \mathrm{Mbps}$, respectively. This indicates that the optimal fair allocation has $50 \%$ higher minimum data rate per user than the hybrid system where the users always connect to both links.

Table 4.7: Indicator variables for each user when implementing the hybrid system where the user always connect to both RF and VLC (Model (3)).

\begin{tabular}{cccccccc} 
& User 1 & User 2 & User 3 & User 4 & User 5 & User 6 & User 7 \\
\hline$I_{R F}$ & 1 & 1 & 1 & 1 & 1 & 1 & 1 \\
$I_{V L C 1}$ & 0 & 1 & 0 & 1 & 0 & 1 & 1 \\
$I_{V L C 2}$ & 1 & 0 & 1 & 0 & 1 & 0 & 0 \\
\hline$R($ Mbps $)$ & 26.79 & 20.83 & 30.56 & 32.68 & 23.05 & 32.78 & 17.07
\end{tabular}

In the case of the hybrid system where the user connects to the best link, Table 4.8 shows the results of implementing the system. The results for the optimal fair allocation shows that not always connecting to the system with strongest power is the best case. The minimum data rate for the proposed system is 25.33 Mbps while connecting to only one system achieved a minimum data rate of $20 \mathrm{Mbps}$, which shows that the proposed system has $26.65 \%$ higher minimum data rate per user.

Table 4.9 summarizes the results for the three hybrid systems. For the optimal fair allocation, the proposed system has $26.65 \%$ and $48.13 \%$ higher minimum data rate than the other two hybrid systems. For the maximum throughput allocation, the proposed system has $26.5 \%$ and $59.6 \%$ higher average data rate than the other two hybrid systems. 
Table 4.8: Indicator variables for each user when implementing the hybrid system where the user connects to either the VLC or RF AP (Model (2)).

\begin{tabular}{cccccccc} 
& User 1 & User 2 & User 3 & User 4 & User 5 & User 6 & User 7 \\
\hline$I_{R F}$ & 0 & 0 & 0 & 1 & 0 & 0 & 1 \\
$I_{V L C 1}$ & 0 & 1 & 0 & 0 & 0 & 1 & 0 \\
$I_{V L C 2}$ & 1 & 0 & 1 & 0 & 1 & 0 & 0 \\
\hline$R(\mathrm{Mbps})$ & 26.67 & 30 & 26.67 & 61.87 & 20 & 50 & 24.75
\end{tabular}

Table 4.9: Comparison between the hybrid systems in term of minimum and average data rate for 7 users.

Optimized min rate Optimized average rate

\begin{tabular}{lcl} 
Model (1): Proposed system & $25.33 \mathrm{Mbps}$ & $43.4 \mathrm{Mbps}$ \\
Model (2): Connected to best system & $20 \mathrm{Mbps}$ & $34.3 \mathrm{Mbps}$ \\
Model (3): Connected to both systems & $17.1 \mathrm{Mbps}$ & $27.2 \mathrm{Mbps}$ \\
\hline
\end{tabular}

Table 4.10 shows the indicator variables for each user when implementing the maximum throughput allocation. As shown in the table, the maximum throughput allocation allows only 3 users to connect to achieve the maximum average data rate. This clearly shows that using the optimization for a dense user environment is a disadvantage for this optimization, as no matter how many users can connect to the system, only the 3 users with the highest data rate will connect to achieve the maximum throughput.

Table 4.10: Indicator variables for each user when implementing the maximum throughput allocation

\begin{tabular}{cccccccc} 
& User 1 & User 2 & User 3 & User 4 & User 5 & User 6 & User 7 \\
\hline$I_{R F}$ & 0 & 0 & 0 & 1 & 0 & 0 & 0 \\
$I_{V L C 1}$ & 0 & 0 & 0 & 0 & 0 & 1 & 0 \\
$I_{V L C 2}$ & 0 & 0 & 1 & 0 & 0 & 0 & 0 \\
\hline$R($ Mbps $)$ & 0 & 0 & 80 & 123.75 & 0 & 100 & 0
\end{tabular}




\section{Chapter 5}

\section{Conclusion and Future Work}

\section{$5.1 \quad$ Summary}

In recent years, research about implementing a hybrid systems to support a higher data rate in RF systems has increased significantly. The use of hybrid VLC and RF systems has shown outstanding results due to the advantages of VLC over RF systems, especially in term of interference and capacity. Many hybrid VLC and RF systems have been proposed recently. In this thesis, the research we performed is summarized as follows:

- We propose a new hybrid system where the users can receive data simultaneously from two different links. The proposed system allows the user to connect to RF and VLC links to achieve one of two goals:

- Fair resource allocation: the maximum possible minimum data rate per user is achieved.

- Maximum throughput allocation: the maximum average data rate is achieved.

- The proposed system is compared with the existing WiFi stand alone system. 
Then we compare it with two other different hybrid systems where the user can connect to both systems at the same time or connect to only one system at a time. We consider VLC systems that either do or do not suffer from adjacentcell interference.

\subsection{Conclusion}

In this thesis, we propose and analyze the use of VLC working in parallel with WiFi to compute the optimal fair allocation or maximum throughput allocation. Simulations show that the proposed system works efficiently for the optimal fair allocation, and the results show outstanding results compared to the other systems. Unlike other hybrid systems, the proposed system supports both low and high user density environment by offloading users from one link to another to achieve the best results. However, applying the maximum throughput allocation seems to be impractical as the proposed system disconnect some users to achieve the best results. Thus, this model should be considered for maximizing the minimum data rate as the simulations for the average data rate when applying the optimal fair allocation shows similar results to the other hybrid systems. This indicates that applying the optimal fair allocation has no penalty on the average data rate.

\subsection{Future Work}

Further studies on the proposed system can be made. One significant area that needs to be considered in future work is the effect of handover in the VLC link on the proposed system. Time delay and energy consumption of the hybrid system might need further studies before implementing the proposed model. Moreover, different schemes for both links, like orthogonal frequency division multiplexing (OFDM) 
should be considered to compute the best results we can achieve. Finally, different algorithms that can handle a large number of users should be addressed. 


\section{Bibliography}

[1] M. M. (2009) VLC electromagnetic spectrum. [Online]. Available: https: //commons.wikimedia.org

[2] J.-Y. Wang, J.-B. Wang, and Y. Wang, "Fundamental analysis for visible light communication with input-dependent noise," in Optical Fiber and Wireless Communications. InTech, 2017.

[3] J. Lian and M. Brandt-Pearce, "Distributed power allocation for multiuser MISO indoor visible light communications," in 2015 IEEE Global Communications Conference (GLOBECOM), Dec 2015, pp. 1-7.

[4] SanderVanGysegem. (2007) schema van een TDMA systeem. [Online]. Available: https://commons.wikimedia.org

[5] K. Technologies. (2007) Concepts of Orthogonal Frequency Division Multiplexing (OFDM) and 802.11 WLAN. [Online]. Available: http://rfmw.em.keysight.com/wireless/helpfiles/89600b/webhelp/ subsystems/wlan-ofdm/Content/ofdm_basicprinciplesoverview.htm

[6] A. Aijaz, H. Aghvami, and M. Amani, "A survey on mobile data offloading: technical and business perspectives," IEEE Wireless Communications, vol. 20, no. 2, pp. 104-112, 2013. 
[7] K. David, D. Dixit, and N. Jefferies, "2020 vision," IEEE Vehicular Technology Magazine, vol. 5, no. 3, pp. 22-29, Sept 2010.

[8] G. R. Hiertz, D. Denteneer, L. Stibor, Y. Zang, X. P. Costa, and B. Walke, "The IEEE 802.11 universe," IEEE Communications Magazine, vol. 48, no. 1, pp. 62-70, January 2010.

[9] M. E. Helou, S. Lahoud, M. Ibrahim, and K. Khawam, "A hybrid approach for radio access technology selection in heterogeneous wireless networks," in European Wireless 2013; 19th European Wireless Conference, April 2013, pp. 1-6.

[10] A. Jovicic, J. Li, and T. Richardson, "Visible light communication: opportunities, challenges and the path to market," IEEE Communications Magazine, vol. 51, no. 12 , pp. 26-32, December 2013.

[11] B. H. Jung, N.-O. Song, and D. K. Sung, "A network-assisted user-centric WiFioffloading model for maximizing per-user throughput in a heterogeneous network," IEEE Transactions on Vehicular Technology, vol. 63, no. 4, pp. 1940$1945,2014$.

[12] Z. Li, C. Dong, A. Li, and H. Wang, "Traffic offloading from LTE-U to WiFi: A multi-objective optimization approach," in Communication Systems (ICCS), 2016 IEEE International Conference on. IEEE, 2016, pp. 1-5.

[13] S. Sagari, I. Seskar, and D. Raychaudhuri, "Modeling the coexistence of LTE and WiFi heterogeneous networks in dense deployment scenarios," in Communication Workshop (ICCW), 2015 IEEE International Conference on. IEEE, 2015, pp. 2301-2306. 
[14] F. Liu, E. Bala, E. Erkip, M. C. Beluri, and R. Yang, "Small-cell traffic balancing over licensed and unlicensed bands," IEEE Transactions on Vehicular Technology, vol. 64, no. 12, pp. 5850-5865, Dec 2015.

[15] W. Guo, Q. Li, H.-y. Yu, and J.-h. Liu, "A parallel transmission MAC protocol in hybrid VLC-RF network," Journal of Communications, vol. 10, no. 1, 2015.

[16] S. Shao, A. Khreishah, M. B. Rahaim, H. Elgala, M. Ayyash, T. D. Little, and J. Wu, "An indoor hybrid WiFi-VLC internet access system," in Mobile Ad Hoc and Sensor Systems (MASS), 2014 IEEE 11th International Conference on. IEEE, 2014, pp. 569-574.

[17] X. Bao, J. Dai, and X. Zhu, "Visible light communications heterogeneous network (VLC-HetNet): new model and protocols for mobile scenario," Wireless Networks, vol. 23, no. 1, pp. 299-309, 2017.

[18] M. Kashef, A. Torky, M. Abdallah, N. Al-Dhahir, and K. Qaraqe, "On the achievable rate of a hybrid PLC/VLC/RF communication system," in Global Communications Conference (GLOBECOM), 2015 IEEE. IEEE, 2015, pp. 16.

[19] Y. Tang, M. Brandt-Pearce, and S. G. Wilson, "Link adaptation for throughput optimization of parallel channels with application to hybrid FSO/RF systems," IEEE Transactions on communications, vol. 60, no. 9, pp. 2723-2732, 2012.

[20] C. Heegard. (2001) Range versus rate in IEEE 802.11g wireless local area networks. [Online]. Available: http://heegard.com/ heegard/papers/RvR.pdf

[21] L. U. Khan, "Visible light communication: Applications, architecture, standardization and research challenges," Digital Communications and Networks, vol. 3, no. 2, pp. 78-88, 2017. 
[22] H. Chowdhury, "Data download on the move in visible light communications: design and analysis." Ph.D. dissertation, University of Oulu, Finland, 2016.

[23] A. M. Cailean, B. Cagneau, L. Chassagne, V. Popa, and M. Dimian, "Evaluation of the noise effects on visible light communications using Manchester and Miller coding," in 2014 International Conference on Development and Application Systems (DAS), May 2014, pp. 85-89.

[24] J. Lian and M. Brandt-Pearce, "Adaptive M-PAM for multiuser MISO indoor VLC systems," in 2016 IEEE Global Communications Conference (GLOBECOM), Dec 2016, pp. 1-6.

[25] A. F. Molisch, Wireless communications. John Wiley \& Sons, 2012, vol. 34.

[26] M. Kashef, M. Abdallah, N. Al-Dhahir, and K. Qaraqe, "On the impact of PLC backhauling in multi-user hybrid VLC/RF communication systems," in Global Communications Conference (GLOBECOM), 2016 IEEE. IEEE, 2016, pp. 1-6. 